\title{
Research on Dual Security Control for a Non-Linear CPS with Multi-Objective Constraints under DoS Attack and Actuator Fault: An Active-Passive Attack-Tolerant Approach
}

\author{
Li Zhao $\mathbb{D}^{1,2}$ Wei Li ${ }^{1}{ }^{1}$, and Yajie $\mathrm{Li}^{1}$ \\ ${ }^{1}$ School of Electronical and Information Engineering, Lanzhou University of Technology, Lanzhou 730050, China \\ ${ }^{2}$ School of Electrical Engineering, Longdong University, Qingyang 745000, China \\ Correspondence should be addressed to Wei Li; liwei@lut.cn
}

Received 16 October 2021; Accepted 8 January 2022; Published 17 February 2022

Academic Editor: Mohamed Hamdy

Copyright (C) $2022 \mathrm{Li}$ Zhao et al. This is an open access article distributed under the Creative Commons Attribution License, which permits unrestricted use, distribution, and reproduction in any medium, provided the original work is properly cited.

Based on the discrete event-triggered communication scheme (DETCS), a codesign method of dual security control and communication for the non-linear cyberphysical system (CPS) with denial-of-service (DoS) attack and actuator fault is studied under multi-objective constraints. First, to effectively distinguish DoS attacks with different energy levels, the DoS attack detection method is established based on the maximum allowable delay of the system. Second, a non-linear CPS dual security control framework that integrates active-passive attack tolerance for DoS attack and active fault tolerance for actuator fault is constructed. Third, the low-energy DoS attack is regarded as a special kind of time delay, and a robust observer for the estimation of system states and actuator fault is designed, and a codesign method of passive attack tolerance, active fault tolerance, and communication is proposed. Furthermore, a correction compensation algorithm of control quantity is proposed for high-energy DoS attack based on the PID idea to make active-passive attack tolerance more effective. Finally, the effectiveness of the proposed method is verified by the simulation of a quadruple-tank model.

\section{Introduction}

With the wide application of the cyber physical system (CPS) in aerospace, intelligent manufacturing, smart grid, and other fields, its security problems have become increasingly prominent. For example, BlackEnergy3 attacked the Ukrainian power grid [1] and Bitcoin Ransomware (WannaCry) attacked global public and commercial systems [2], which have caused immeasurable losses to national security and economy [3]. In general, CPS insecurity factors mainly come from two aspects. First, the communication network becomes open and interconnected from the traditional closed and isolated, which makes it more vulnerable to various types of cyber attacks and indirectly affects the security of physical plants or even the whole system $[4,5]$. Second, the physical components operate under high load or in harsh environment for a long time, resulting in the degradation of core components such as fail of actuators, thus destroying the stability of the system $[6,7]$. In addition, most of the actual systems have non-linear characteristic, so it is important to study the security control for a non-linear CPS.

Cyber attacks on the CPS mainly include denial-ofservice (DoS) $[8,9]$ and false data injection (FDI) attack $[10,11]$. DoS attacks are non-stealthy attacks that often block communication channels and make system communication impossible [12], and the Ukraine power grid incident was caused by using DoS attacks to block communication transmission. Scholars usually model DoS attacks in the CPS as network time delay or packet loss [13-16]. In [17], DoS attack is modeled as bounded packet loss by defining attack frequency and duration. In [18], the packet loss is regarded as a random Bernoulli distribution. In [19], DoS attack is modeled as a Markov process. After the attack model is established, the methods based on control theory are further used to ensure the system security, such as state estimation 
$[20,21]$, game theory $[22,23]$, predictive control $[24,25]$, and resilient control $[26,27]$, among which the resilient control has fruitful achievements and it is also the focus of attention. In [28], the asynchronous attack signal is described by recursive characteristics, and a dynamic eventtriggered resilient control method is proposed to resist DoS attack. In [29], a resilient control strategy is studied under DoS attack, and furthermore, an $H_{\infty}$ observer is constructed based on the periodic event-triggered control framework. The resilient control problem is studied in [30] based on the sampling data model under DoS attacks, and in order to receive the sampling signal and obtain the attack duration, a logic processor is introduced into the controller. The defense against DoS attacks in that mentioned above is essentially robust to packet loss or time delay caused by finite-energy DoS attacks, and it is a passive attack-tolerant strategy that can only cope with low-energy DoS attacks. Once the packet loss or delay caused by a DoS attack exceeds the maximum allowable range, the system security will not be guaranteed. The active attack-tolerant method can be further used to reconstruct or correct the loss of control quantity caused by high-energy DoS attack, i.e., the combination of active and passive attack tolerance will better guarantee the CPS security. This is one of the motivations in this paper.

Although there are many results to deal with DoS attack in the CPS, there is a lack of common consideration of DoS attack and physical system fault. In [31-33], passive, active, and active-passive hybrid fault-tolerant control methods are proposed, respectively, to suppress the impact of faults on the system. In [34], a novel dynamic fault-tolerant control model is proposed. Therefore, the fault-tolerant control provides an effective solution to component faults. However, the impact of DoS attack on the system is not considered in that mentioned above. How to take into account the attack tolerance in a cyber system and the fault tolerance in a physical system and carry out the codesign of dual security control is undoubtedly of great significance for the improvement of CPS security. This is another motivation for the research.

The CPS is the integration of the cyber and physical system, and a network communication scheme is important for the coordination of control and communication. The traditional time-triggered communication scheme not only wastes network resources but also the design of the control strategy lacks proper correlation with the communication [35]. The discrete event-triggered communication scheme (DETCS) proposed in $[36,37]$ relies on "events" rather than "time" for data transmission, which can save network resources and make the collaboration of control and communication possible. Recently, the adaptive event-triggered communication scheme proposed in $[38,39]$ is able to satisfy the control requirements while saving network resources more effectively, but it is still difficult to achieve the coordination of security control and communication under DoS attack. Besides, the practical engineering has other performance requirements in addition to security. The T-S fuzzy model is a powerful method for the analysis and synthesis of a non-linear system [40]; therefore, based on the T-S fuzzy model and DETCS, it is more challenging and valuable to study the codesign of dual security control and communication for a non-linear CPS with DoS attacks and actuator fault under multi-objective constraints. This is the third research motivation of this study.

Based on previous studies, in this paper, the codesign method of dual security control and communication for a non-linear CPS with DoS attacks and actuator fault is investigated. The contributions are as follows:

(1) A DoS attack detection method is proposed based on the maximum allowable delay of the system to distinguish high- and low-energy DoS attacks, and a passive attack-tolerance strategy is used for lowenergy DoS attack. Based on the PID idea, the active compensation strategy is designed for the lack of control quantity caused by high-energy DoS attacks, the combination of active and passive attack tolerance strategies can more effectively resist the impact of DoS attacks on the CPS.

(2) A dual security control framework with activepassive attack tolerance and active fault tolerance is constructed for the coexistence of DoS attacks and actuator fault in the CPS. Furthermore, a non-linear CPS closed-loop T-S fuzzy model integrating DoS attack, actuator fault, and attack-tolerant and faulttolerant control is established, which lays a foundation for the dual security control.

(3) Based on the DETCS, considering the multi-objective constraints, such as $\alpha$-stability and $H_{\infty}$ performance, the design methods of the observer for state and fault and dual security controller are given, respectively, with the help of less-conservative techniques. Finally, the codesign between dual security control and communication resource saving is realized, and a classical quadruple-tank model is used to verify the effectiveness of the proposed method.

The remainder of this paper is organized as follows. The system description is presented in Section 2. In Section 3, an observer for system state and actuator fault with multiobjective constraints under DoS attack is designed. The dual security controller is presented in Section 4 . The strategy and algorithm of DoS attack detection and active attack tolerance are given in Section 5. Section 6 is the simulation research and result analysis, and the conclusion is presented in Section 7.

\section{System Description}

2.1. System Framework. In order to keep the non-linear CPS which suffers from DoS attack and continuous time-varying actuator fault stable, a dual security control framework with active-passive attack tolerance and active fault tolerance is established, as shown in Figure 1.

In Figure 1, the system consists of a non-linear controlled plant, an intelligent sensing unit, a control unit, an intelligent execution unit, and dual-end networks.

The intelligent sensing unit is composed of a sensor, a sampler, an observer, and an event generator. The control quantity and system output are measured by using a sensor, 


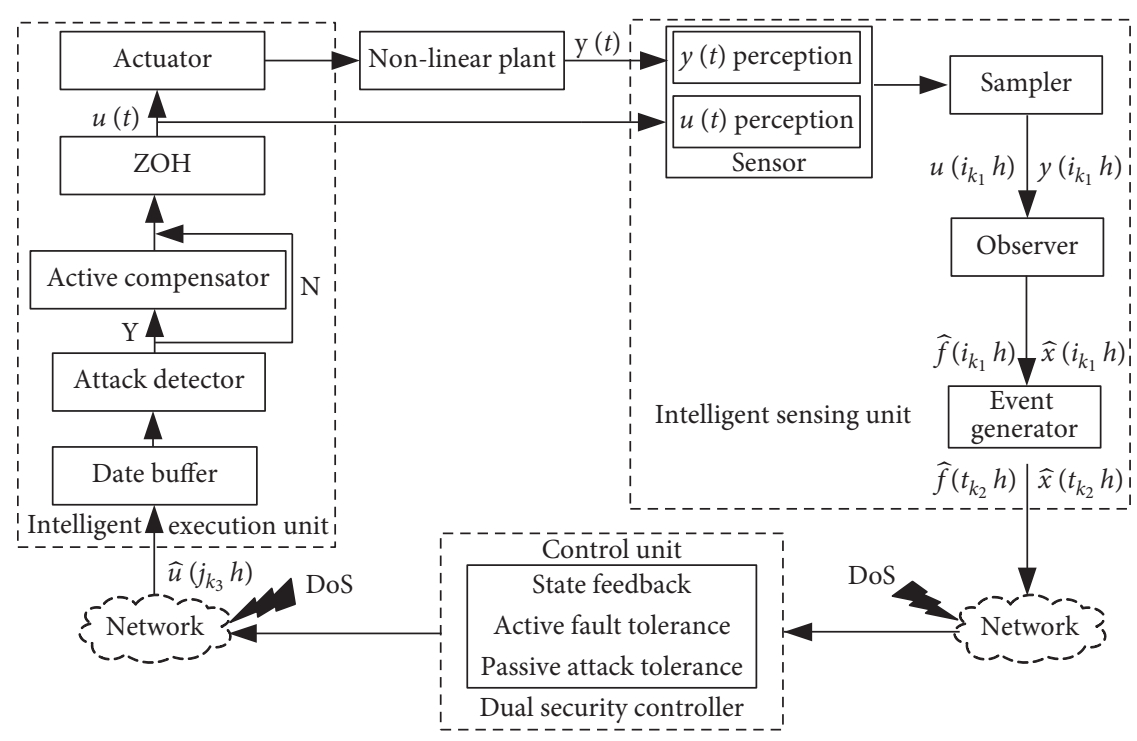

FIgURE 1: Dual security control framework for a non-linear CPS under DoS attack.

the measured values are sampled by using a sampler in an equal periodic manner, and the sampled data are observed by the observer to obtain the estimations of system state and fault. After screening by using the event generator, the data that meet the trigger condition are transmitted to the control unit through the sensor network in a non-uniform manner.

The control unit includes a dual security controller with passive attack tolerance and active fault tolerance. It mainly completes the calculation of control quantity based on resilient control for DoS attack with low energy and actuator fault, and then, the calculated quantity is transmitted to the execution unit through the control network. Obviously, the calculation of control quantity is also carried out in a nonuniform periodic manner.

The intelligent execution unit includes a data buffer, an attack detector, an attack compensator, a zero-order holder $(\mathrm{ZOH})$, and an actuator. The data buffer stores the received control quantity and further implements attack detection. If it is detected that the time delay caused by DoS attack is greater than the maximum allowed value, then it is a highenergy DoS attack. It will be updated and corrected with the help of appropriate compensation algorithm according to the original value of control quantity stored in the early stage. It is further sent to the actuator to achieve active attack tolerance for high-energy DoS attacks. If the time delay is less than the maximum allowed value, it is a low-energy DoS attack, the received data will be directly sent to the actuator, and finally, the control quantity will act on the controlled plant.

Remark 1: if the time delay caused by DoS attack approaches or exceeds the maximum allowable delay of the system, the attack is called high-energy DoS attack; otherwise, it is called low-energy DoS attack.

Remark 2: the passive attack-tolerant strategy may fail when the system suffers from high-energy DoS attack.
At this time, to ensure the system is stable, the active attack-tolerant strategy is implemented to compensate and update the loss of control quantity caused by highenergy DoS attack, so the system has the ability of active-passive attack-tolerant control.

Remark 3: according to the characteristic of DoS attack, it can be regarded as a special packet loss and transformed into time delay through the following timing analysis. Based on the maximum allowable delay of the system, the DoS attack detection method is proposed. If the time delay caused by the DoS attack is within the allowable range, the passive attack tolerance is used. On the contrary, the active attack-tolerant strategy is used to compensate the loss of control quantity caused by the DoS attack. Finally, the system remains stable under both high- and low-energy DoS attacks.

Assumption 1. The energy of DoS attack is limited, and its impact on the system is regarded as a special kind of packet loss, and the amount of packets lost is limited.

Assumption 2. Natural packet loss rarely occurs during data transmission, and a single packet with time stamp is used for data transmission.

2.2. Description of the Controlled Plant. As shown in Figure 1, it can be seen that the output of the continuous non-linear controlled plant is sampled and transmitted to the intelligent sensing unit and control unit, and then, both estimation and calculation are digital quantities. Therefore, the system is a typical sampling data system [41]. Based on the T-S fuzzy model, the non-linear CPS with actuator continuous time-varying fault is modeled as follows: 


$$
\left\{\begin{array}{l}
\dot{x}(t)=\sum_{i=1}^{r} \xi_{i}(\theta(t))\left[\mathbf{A}_{i} \mathbf{x}(t)+\mathbf{B}_{i} \mathbf{u}(t)+\mathbf{E}_{f i} \mathbf{f}(t)+\mathbf{E}_{w i} \mathbf{w}(t)\right] \\
\mathbf{y}\left(i_{k} h\right)=\sum_{i=1}^{r} \xi_{i}(\theta(t))\left[\mathbf{C}_{i} \mathbf{x}\left(i_{k} h\right)+\mathbf{E}_{v i} \mathbf{v}\left(i_{k} h\right)\right]
\end{array}\right.
$$

where $\xi_{i}(\theta(t))=a_{i}(\theta(t)) / \sum_{i=1}^{N} a_{i}(\theta(t)), \xi_{i}(\theta(t))$ is the weight ratio representing each fuzzy rule, $a_{i}(\theta(t))=\prod_{j=1}^{N} \mathbf{M}_{i j}\left(\theta_{j}(t)\right), \mathbf{M}_{i j}\left(\theta_{j}(t)\right)$ is the membership function of $\theta_{j}(t)$ with respect to $\mathbf{M}_{i j}$, let $a_{i}(\theta(t)) \geq 0(i=$ $1,2, \cdots, N)$ and $\sum_{i=1}^{N} a_{i}(\theta(t))>0$, and then, $\xi_{i}(\theta(t)) \geq 0$ and $\sum_{i=1}^{N} \xi_{i}(\theta(t))=1 . \mathbf{A}_{i}, \mathbf{B}_{i}, \mathbf{E}_{f i}, \mathbf{E}_{w i}, \mathbf{C}_{i}, \mathbf{E}_{v i}$ are matrices with known appropriate dimensions, and $\mathbf{x}(t) \in R^{n}, \mathbf{u}(t) \in R^{n_{u}}$ are the system state and the control input vector, respectively. $\mathbf{f}(t) \in R^{n_{f}}$ is a continuous time-varying actuator fault, and its derivative is assumed to be bounded; i.e., there is a constant $f_{1}$ such that $\|\dot{f}(t)\| \leq f_{1} . \mathbf{w}(t) \in R^{n_{w}}, \mathbf{y}\left(i_{k} h\right) \in R^{m}$, and $\mathbf{v}\left(i_{k} h\right) \in R^{n_{v}}$ are the system disturbance, output sampling, and measurement noise.

In order to effectively save network resources and realize the codesign of communication and control, the following classic DETCS [36] is adopted:

$$
\mathbf{e}^{T}\left(i_{k_{1}} h\right) \boldsymbol{\Phi}\left(i_{k_{1}} h\right) \leq \sigma_{s} \widehat{x}^{T}\left(t_{k_{2}} h\right) \Phi \widehat{x}\left(t_{k_{2}} h\right) .
$$

In the trigger condition equation (2), the trigger parameter $\sigma_{s}$ is given in advance, $\delta_{s} \in[0,1)$, and it is related to the expected performance of the system. The positive definite symmetric matrix $\Phi$ is to be designed. $\mathbf{e}\left(i_{k_{1}} h\right)=\hat{x}\left(i_{k_{1}} h\right)-\hat{x}\left(t_{k_{2}} h\right)$ is the state estimation error, $\widehat{x}\left(i_{k_{1}} h\right)$ is the system state estimation at the current time, and $\widehat{x}\left(t_{k_{2}} h\right)$ is the system state estimation that meets the event trigger condition at the previous time.

Assumption 3. The sampler is clock driven in an equal period, the sampling period is $h$, and the corresponding sequence is $\left\{i_{k_{1}}\right\}, k_{1}=0,1,2, \cdots$. The controller and actuator are event driven, and the filtered data trigger period is recorded as $h_{t_{k_{2}}}, h_{t_{k_{2}}}=\left(t_{k_{2}+1}-t_{k_{2}}\right) h$, and the transmission sequence is $\left\{t_{k_{2}}\right\}, k_{2}=0,1,2, \ldots$ After the DoS attack invasion, the period of successful transmission of the filtered data by the event generator is $h_{j_{k_{3}}}, h_{j_{k_{3}}}=\left(j_{k_{3}+1}-j_{k_{3}}\right) h$, the transmission sequence is $\left\{j_{k_{3}}\right\}, k_{3}=0,1,2, \ldots$, and the interval satisfies $t_{k_{2}}=m i_{k_{1}}, j_{k_{3}}=n i_{k_{1}}$, where $m, n$ are integers.

2.3. Analysis of Time-Delay Interval. Although the controlled plant is continuous, the calculation of the observer and controller is carried out in the form of digital quantity. In addition, the introduction of the DETCS makes the filtered data transmitted in a non-uniform manner. Based on the time-delay system theory method in [42], the non-uniform transmission property is transformed into time delay for the typical sampling data system, and the design method of observer and controller are studied in a continuous manner.
The output sampling of the intelligent sensing unit is carried out in an equal periodic manner, and its period is $h$. With the help of time-delay system theory, the sampling period in adjacent sampling points is transformed into time delay, and then, the system is designed and analyzed in a continuous manner.

The delay function is defined as

$$
\tau_{1}(t)=t-i_{k_{1}} h,
$$

where $t \in\left[i_{k_{1}} h, i_{k_{1}+1} h\right)$ and $0<\tau_{1}(t) \leq h_{1}=h$. The time sequence of the sampled, filtered, and transmitted data is shown in Figure 2.

As shown in Figure 2, the amount of consecutive packet losses caused by DoS attack is $\tau_{t_{k_{2}}}^{D o S} \in\left[0, \tau_{M}^{D o S}\right] . h_{t_{k_{2}}}^{\max }$ is the non-uniform maximum trigger period, and then, the actual maximum duration of DoS attack is $\tau_{M}^{D o s} h_{t_{k_{2}}}^{\max } . i_{k_{1}} h$ is the sampled data sequence, and $t_{k_{2}} h$ is the triggered data sequence. $j_{k_{3}} h$ is the transmitted sequence to the $\mathrm{ZOH}$ (without considering transmission delay), and it satisfies the relationship $j_{k_{3}} h=t_{k_{2}} h+\tau_{t_{k_{2}}}^{D o s} h_{t_{k_{2}}}$.

When $\widehat{x}\left(j_{k_{3}} h\right)$ and $\hat{f}\left(j_{k_{3}} h\right)$ are transmitted to the front end of the $\mathrm{ZOH}$, while $\widehat{x}\left(j_{k_{3}+1} h\right)$ and $\hat{f}\left(j_{k_{3}+1} h\right)$ are not sent to the $\mathrm{ZOH}$, the transmission interval is $\Lambda=\left[j_{k_{3}} h, j_{k_{3}+1} h\right)$.

The delay function is defined as

$$
\tau_{2}(t)=t-j_{k_{3}} h .
$$

Then, its upper bound is

$$
h_{2}=\max \left\{h_{t_{k_{2}}}+\tau_{t_{k_{2}}}^{D o s} h_{t_{k_{2}}}\right\}=h_{t_{k_{2}}}^{\max }+\tau_{M}^{D o s} h_{t_{k_{2}}}^{\max } .
$$

The lower bound is $0<\tau_{\min }=\min \left\{\tau_{t_{k_{2}}}^{D o s} h_{t_{k_{2}}}\right\}$, where the time-delay function satisfies $0<\tau_{\min } \leq \tau_{2}(t) \leq h_{2}$. For the convenience of analysis, $i_{k_{1}} h, t_{k_{2}} h$, and $j_{k_{3}} h$ will be recorded as $i_{k_{1}}, t_{k_{2}}$, and $j_{k_{3}}$.

\section{Observer Design for System State and Actuator Fault with Multi-Objective Constraints under DoS Attack}

Design objective: under the DETCS, considering DoS attack and actuator fault, the designed observer can accurately estimate the system state and actuator fault in real time with $\alpha$ - stability and $H_{\infty}$ performance.

According to the dual security control framework for a non-linear CPS in Figure 1 and the analysis in Section 2.3, the output characteristics of the system are considered as time delay in one sampling period [43], and the system output is obtained as follows by combining with equations (1) and (3):

$$
\mathbf{y}(t)=\sum_{i=1}^{r} \xi_{i}(\theta(t))\left[\mathbf{C}_{i} \mathbf{x}\left(t-\tau_{1}(t)\right)+\mathbf{E}_{v i} \mathbf{v}\left(t-\tau_{1}(t)\right)\right] .
$$

Constructing an observer for the estimation of state and fault, 


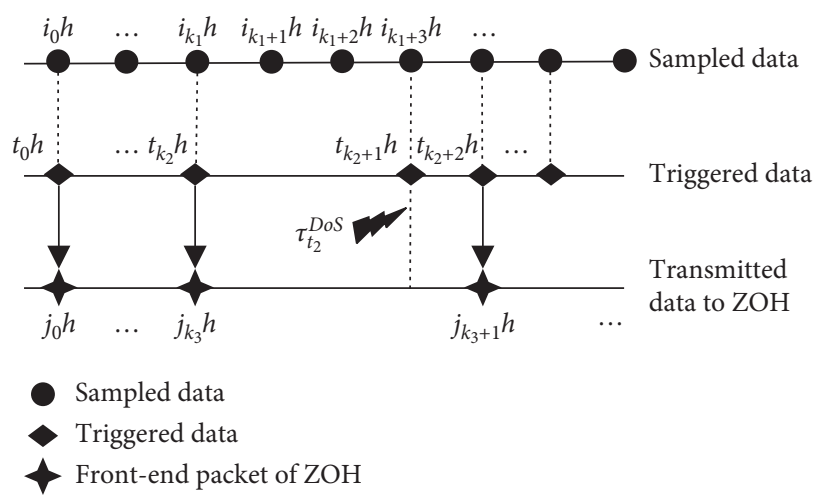

FIGURE 2: Time sequence of updated data for a non-linear CPS under DoS attack.

$$
\left\{\begin{array}{l}
\dot{\hat{x}}(t)=\sum_{i=1}^{r} \sum_{j=1}^{r} \xi_{i}(\theta(t)) \xi_{j}(\theta(t))\left[\mathbf{A}_{i} \widehat{x}(t)+\mathbf{B}_{i} \mathbf{u}(t)+\mathbf{E}_{\mathbf{f} i} \widehat{f}(t)-\mathbf{L}_{j}(\widehat{y}(t)-\mathbf{y}(t))\right] \\
\hat{y}(t)=\sum_{i=1}^{r} \xi_{i}(\theta(t))\left[\mathbf{C}_{i} \hat{x}\left(t-\tau_{1}(t)\right)\right] \\
\dot{\hat{f}}(t)=\sum_{i=1}^{r} \xi_{i}(\theta(t))\left[-\mathbf{F}_{j} \mathbf{e}_{y}(t)\right]
\end{array}\right.
$$

where $\hat{x}(t), \hat{y}(t), \hat{f}(t)$ represent the estimations of system state, observer output, and fault, respectively, and $\mathbf{L}_{j}, \mathbf{F}_{j}$ are the observer and fault estimation gain matrix.
We define $\mathbf{e}_{x}(t)=\widehat{x}(t)-\mathbf{x}(t), \mathbf{e}_{y}(t)=\hat{y}(t)-\mathbf{y}(t)$, and $\mathbf{e}_{f}(t)=\hat{f}(t)-\mathbf{f}(t)$, and the following error system is obtained:

$$
\left\{\begin{array}{l}
\dot{e}_{x}(t)=\dot{\hat{x}}(t)-\dot{x}(t)=\sum_{i=1}^{r} \sum_{j=1}^{r} \xi_{i}(\theta(t)) \xi_{j}(\theta(t))\left[\mathbf{A}_{i} \mathbf{e}_{x}(t)+\mathbf{E}_{\mathbf{f} i} \mathbf{e}_{f}(t)-\mathbf{L}_{j} \mathbf{C}_{i} \mathbf{e}_{x}\left(t-\tau_{1}(t)\right)+\mathbf{L}_{j} \mathbf{E}_{\mathbf{v} i}\left(t-\tau_{1}(t)\right)-\mathbf{E}_{\mathbf{w} i} \mathbf{w}(t)\right] \\
\mathbf{e}_{y}(t)=\sum_{i=1}^{r} \xi_{i}(\theta(t))\left[\mathbf{C}_{i} \mathbf{e}_{x}\left(t-\tau_{1}(t)\right)-\mathbf{E}_{\mathbf{v} i} \mathbf{v}\left(t-\tau_{1}(t)\right)\right] .
\end{array}\right.
$$

The derivative of the fault estimation error with respect to time is

$$
\begin{aligned}
\dot{e}_{f}(t)= & \sum_{i=1}^{r} \sum_{j=1}^{r} \xi_{i}(\theta(t)) \xi_{j}(\theta(t))\left[-\mathbf{F}_{j} \mathbf{C}_{i} \mathbf{e}_{x}\left(t-\tau_{1}(t)\right)\right. \\
& \left.+\mathbf{F}_{j} \mathbf{E}_{\mathbf{v} i} \mathbf{v}\left(t-\tau_{1}(t)\right)-\dot{f}(t)\right]
\end{aligned}
$$

Equations (8) and (9) are augmented to the following system:

$$
\begin{aligned}
\dot{\bar{e}}(t)= & \sum_{i=1}^{r} \sum_{j=1}^{r} \xi_{i}(\theta(t)) \xi_{j}(\theta(t))\left[\bar{A}_{i} \bar{e}(t)-\bar{E}_{\mathbf{w i}} \bar{w}(t)\right. \\
& \left.-\bar{L}_{j} \bar{C}_{i} \bar{e}\left(t-\tau_{1}(t)\right)+\bar{L}_{j} \mathbf{E}_{\mathbf{v} i} \mathbf{v}\left(t-\tau_{1}(t)\right)\right],
\end{aligned}
$$

where

$$
\begin{aligned}
\bar{A}_{i} & =\left[\begin{array}{cc}
\mathbf{A}_{i} & \mathbf{E}_{\mathbf{f} i} \\
\mathbf{0} & \mathbf{0}
\end{array}\right], \\
\bar{e}(t) & =\left[\begin{array}{c}
\mathbf{e}_{x}(t) \\
\mathbf{e}_{f}(t)
\end{array}\right], \\
\bar{C}_{i} & =\left[\begin{array}{ll}
\mathbf{C}_{i} & \mathbf{0}
\end{array}\right], \\
\bar{L}_{j} & =\left[\begin{array}{c}
\mathbf{L}_{j} \\
\mathbf{F}_{j}
\end{array}\right], \\
\bar{E}_{\mathbf{w} i} & =\left[\begin{array}{cc}
\mathbf{E}_{\mathbf{w} i} & \mathbf{0} \\
\mathbf{0} & \mathbf{I}
\end{array}\right], \\
\bar{w}(t) & =\left[\begin{array}{c}
\mathbf{w}(t) \\
\dot{f}(t)
\end{array}\right] .
\end{aligned}
$$

Theorem 1. Considering the augmented error system in equation (10) with actuator fault and DoS attacks, given positive numbers $\alpha_{1}, h_{1}$, and $\gamma_{1}, n_{i}, i=1,2,3$, if there exist 
symmetric matrix $\mathbf{P}>\mathbf{0}$ and appropriate dimensional matrices $\mathbf{X}, \mathbf{Y}$, the following matrix inequalities hold:

$$
\left[\begin{array}{ccccc}
\Gamma_{11} & \Gamma_{12} & \Gamma_{13} & \Gamma_{14} & \mathbf{0} \\
* & \Gamma_{22} & \Gamma_{23} & \Gamma_{24} & h_{1} n_{1} e^{2 \alpha_{1} \tau_{1}(t)} \bar{C}_{i}^{\mathrm{T}} \mathbf{Y}_{j}^{\mathrm{T}} \\
* & * & \Gamma_{33} & \Gamma_{34} & \mathbf{0} \\
* & * & * & \Gamma_{44} & \mathbf{0} \\
* & * & * & * & -h_{1} n_{1} e^{2 \alpha_{1} \tau_{1}(t)} \mathbf{P}
\end{array}\right]<0,
$$$$
\left[\begin{array}{ccccc}
\Gamma_{11}^{\prime} & \Gamma_{12}^{\prime} & \Gamma_{13} & \Gamma_{14} & \mathbf{X} \\
* & \Gamma_{22}^{\prime} & \Gamma_{23} & \Gamma_{24} & \mathbf{X} \\
* & * & \Gamma_{33} & \Gamma_{34} & \mathbf{X} \\
* & * & * & \Gamma_{44} & \mathbf{X} \\
* & * & * & * & -\frac{15 n_{1}}{23 h_{1}} \mathbf{P}
\end{array}\right]<0,
$$$$
\left[\begin{array}{ccccccc}
\Lambda_{11} & \Lambda_{12} & \Lambda_{13} & \Lambda_{14} & \Lambda_{15} & \Lambda_{16} & 0 \\
* & \Lambda_{22} & \Lambda_{23} & \Lambda_{24} & \Lambda_{25} & \Lambda_{26} & \Lambda_{27} \\
* & * & \Lambda_{33} & \Lambda_{34} & \mathbf{0} & \mathbf{0} & \mathbf{0} \\
* & * & * & \Lambda_{44} & \mathbf{0} & \mathbf{0} & \mathbf{0} \\
* & * & * & * & \Lambda_{55} & \Lambda_{56} & \Lambda_{57} \\
* & * & * & * & * & \Lambda_{66} & \mathbf{0} \\
* & * & * & * & * & * & \Lambda_{77}
\end{array}\right]<0,
$$$$
\left[\begin{array}{ccccccc}
\boldsymbol{\Lambda}_{11}^{\prime} & \boldsymbol{\Lambda}_{12}^{\prime} & \boldsymbol{\Lambda}_{13}^{\prime} & \boldsymbol{\Lambda}_{14}^{\prime} & \boldsymbol{\Lambda}_{15}^{\prime} & \boldsymbol{\Lambda}_{16}^{\prime} & \mathbf{X} \\
* & \boldsymbol{\Lambda}_{22}^{\prime} & \boldsymbol{\Lambda}_{23}^{\prime} & \boldsymbol{\Lambda}_{24}^{\prime} & \mathbf{0} & \mathbf{0} & \mathbf{X} \\
* & * & \boldsymbol{\Lambda}_{33}^{\prime} & \boldsymbol{\Lambda}_{34}^{\prime} & \mathbf{0} & \mathbf{0} & \mathbf{X} \\
* & * & * & \boldsymbol{\Lambda}_{44}^{\prime} & \mathbf{0} & \mathbf{0} & \mathbf{X} \\
* & * & * & * & \boldsymbol{\Lambda}_{55}^{\prime} & \mathbf{0} & \mathbf{0} \\
* & * & * & * & * & \boldsymbol{\Lambda}_{66}^{\prime} & \mathbf{0} \\
* & * & * & * & * & * & \boldsymbol{\Lambda}_{77}^{\prime}
\end{array}\right]<0 .
$$

Then, the system equation (10) has $\alpha$ - stability and meets the following $\mathbf{H}_{\infty}$ performance index:

$\left\|\bar{e}_{\alpha_{1}}(t)\right\|_{2}^{2} \leq \gamma_{1}^{2}\left[\left\|\bar{w}_{\alpha_{1}}(t)\right\|_{2}^{2}+\sum_{k_{1}=0}^{+\infty}\left(i_{k_{1}+1}-i_{k_{1}}\right)\left\|\mathbf{v}_{\alpha_{1}}\left(i_{k_{1}}\right)\right\|_{2}^{2}\right]$. where

The gain matrix $\mathbf{L}_{j}$ and $\mathbf{F}_{j}$ can be obtained by $\bar{L}_{j}=\left[\begin{array}{l}\mathbf{L}_{j} \\ \mathbf{F}_{j}\end{array}\right]$

$$
\begin{aligned}
\Gamma_{11}= & \mathbf{P} \bar{A}_{i}+\bar{A}_{i}^{T} \mathbf{P}-n_{2} \mathbf{P}-3 \mathbf{X}-3 \mathbf{X}^{T}+h_{1} n_{1} \bar{A}_{i}^{T} \mathbf{P} \bar{A}_{i} \\
& +h_{1} n_{2}\left(\mathbf{P} \bar{A}_{i}+\bar{A}_{i}^{T} \mathbf{P}\right)
\end{aligned}
$$

$$
\begin{aligned}
\Gamma_{13}= & 2 \mathbf{X}-3 \mathbf{X}^{T}, \\
\Gamma_{14}= & 6 \mathbf{X}-3 \mathbf{X}^{T}, \\
\Gamma_{12}= & -e^{\alpha_{1} \tau_{1}(t)} \mathbf{Y}_{j} \bar{C}_{i}+n_{2} \mathbf{P}+\mathbf{X}-3 \mathbf{X}^{T}-h_{1} n_{2} \bar{A}_{i}^{T} \mathbf{P} \\
& -h_{1} n_{2} e^{\alpha_{1} \tau_{1}(t)} \mathbf{Y}_{j} \bar{C}_{i}-h_{1} n_{1} e^{\alpha_{1} \tau_{1}(t)} \bar{A}_{i}^{T} \mathbf{Y}_{j} \bar{C}_{i}, \\
\Gamma_{22}= & h_{1} n_{3} \mathbf{P}-n_{2} \mathbf{P}+\mathbf{X}+\mathbf{X}^{T}+h_{1} n_{2} e^{\alpha_{1} \tau_{1}(t)} \mathbf{Y}_{j} \bar{C}_{i} \\
& +h_{1} n_{2} e^{\alpha_{1} \tau_{1}(t)} \bar{C}_{i}^{T} \mathbf{Y}_{j}^{T}, \\
\Gamma_{23}= & 2 \mathbf{X}+\mathbf{X}^{T}, \\
\Gamma_{24}= & 6 \mathbf{X}+\mathbf{X}^{T}, \\
\Gamma_{33}= & 2 \mathbf{X}+2 \mathbf{X}^{T}, \\
\Gamma_{34}= & 6 \mathbf{X}+2 \mathbf{X}^{T}, \\
\Gamma_{44}= & 6 \mathbf{X}+6 \mathbf{X}^{T}, \\
\Gamma_{11}^{\prime}= & \mathbf{P} \bar{A}_{i}+\bar{A}_{i}^{T} \mathbf{P}-n_{2} \mathbf{P}-3 \mathbf{X}-3 \mathbf{X}^{T}, \\
\Gamma_{12}^{\prime}= & -e^{\alpha_{1} \tau_{1}(t)} \mathbf{Y}_{j} \bar{C}_{i}+n_{2} \mathbf{P}+\mathbf{X}-3 \mathbf{X}^{T}, \\
\Gamma_{22}^{\prime}= & -h_{1} n_{3} \mathbf{P}-n_{2} \mathbf{P}+\mathbf{X}+\mathbf{X}^{T}, \\
\Lambda_{11}= & \mathbf{P} \bar{A}_{i}+\bar{A}_{i}^{T} \mathbf{P}-n_{2} \mathbf{P}-3 \mathbf{X}-3 \mathbf{X}^{T}+\mathbf{I}+h_{1} n_{1} \bar{A}_{i}^{T} \mathbf{P} \bar{A}_{i} \\
& +h_{1} n_{2}\left(\mathbf{P} \bar{A}_{i}+\bar{A}_{i}^{T} \mathbf{P}\right),
\end{aligned}
$$

$\Lambda_{12}=\Gamma_{12}$,

$\Lambda_{13}=\Gamma_{13}$,

$\Lambda_{14}=\Gamma_{14}$,

$\boldsymbol{\Lambda}_{15}=e^{\alpha_{1} \tau_{1}(t)} \mathbf{Y}_{j} \mathbf{E}_{\mathbf{v} i}+h_{1} n_{1} e^{\alpha_{1} \tau_{1}(t)} \bar{A}_{i}^{T} \mathbf{Y}_{j} \mathbf{E}_{\mathbf{v} i}$ $+h_{1} n_{2} e^{\alpha_{1} \tau_{1}(t)} \mathbf{Y}_{j} \mathbf{E}_{\mathbf{v} i}$,

$\Lambda_{16}=-\mathbf{P} \bar{E}_{\mathbf{w} i}-h_{1} n_{1} \bar{A}_{i}^{T} \mathbf{P} \bar{E}_{\mathbf{w} i}-h_{1} n_{2} \mathbf{P} \bar{E}_{\mathbf{w} i}$,

$\Lambda_{22}=\Gamma_{22}$,

$\Lambda_{23}=\Gamma_{23}$,

$\Lambda_{24}=\Gamma_{24}$,

$\boldsymbol{\Lambda}_{25}=-h_{1} n_{2} e^{\alpha_{1} \tau_{1}(t)} \mathbf{Y}_{j} \mathbf{E}_{\mathbf{v} i}$,

$\boldsymbol{\Lambda}_{26}=h_{1} n_{1} e^{\alpha_{1} \tau_{1}(t)} \bar{C}_{i}^{T} \mathbf{Y}_{j}^{T} \bar{E}_{\mathbf{w} i}+h_{1} n_{2} \mathbf{P} \bar{E}_{\mathbf{w} i}$,

$\boldsymbol{\Lambda}_{55}=-\gamma_{1}^{2} \mathbf{I}$,

$\Lambda_{27}=h_{1} n_{1} e^{2 \alpha_{1} \tau_{1}(t)} \bar{C}_{i}^{T} \mathbf{Y}_{j}^{T}$

$\Lambda_{33}=\Gamma_{33}$,

$\Lambda_{34}=\Gamma_{34}$,

$\boldsymbol{\Lambda}_{44}=\Gamma_{44}$,

$\boldsymbol{\Lambda}_{56}=-h_{1} n_{1} e^{\alpha_{1} \tau_{1}(t)} \mathbf{E}_{\mathbf{v} i}^{T} \mathbf{Y}_{j}^{T} \bar{E}_{\mathbf{w} i}$,

$\boldsymbol{\Lambda}_{57}=h_{1} n_{1} e^{2 \alpha_{1} \tau_{1}(t)} \mathbf{E}_{\mathbf{v} i}^{T} \mathbf{Y}_{j}^{T}$,

$\boldsymbol{\Lambda}_{66}=-\gamma_{1}^{2} \mathbf{I}+h_{1} n_{1} \bar{E}_{\mathbf{w i}}^{T} \mathbf{P} \bar{E}_{\mathbf{w i}}$,

$\boldsymbol{\Lambda}_{77}=-h_{1} n_{1} e^{2 \alpha_{1} \tau_{1}(t)} \mathbf{P}$, 


$$
\begin{aligned}
\boldsymbol{\Lambda}_{11}^{\prime} & =\Gamma_{11}^{\prime}+\mathbf{I}, \\
\boldsymbol{\Lambda}_{12}^{\prime} & =\Gamma_{12}^{\prime}, \\
\boldsymbol{\Lambda}_{13}^{\prime} & =\Gamma_{13}, \\
\boldsymbol{\Lambda}_{14}^{\prime} & =\Gamma_{14}, \\
\boldsymbol{\Lambda}_{15}^{\prime} & =e^{\alpha_{1} \tau_{1}(t)} \mathbf{Y} \mathbf{E}_{\mathbf{v} i}, \\
\boldsymbol{\Lambda}_{16}^{\prime} & =-\mathbf{P} \bar{E}_{\mathbf{w} i}, \\
\boldsymbol{\Lambda}_{23}^{\prime} & =\Gamma_{23}, \\
\boldsymbol{\Lambda}_{24}^{\prime} & =\Gamma_{24}, \\
\boldsymbol{\Lambda}_{22}^{\prime} & =-h_{1} n_{3} \mathbf{P}-n_{2} \mathbf{P}+\mathbf{X}+\mathbf{X}^{T}, \\
\boldsymbol{\Lambda}_{33}^{\prime} & =\Gamma_{33}, \\
\Lambda_{34}^{\prime} & =\Gamma_{34}, \\
\Lambda_{44}^{\prime} & =\Gamma_{44}, \\
\Lambda_{55}^{\prime} & =-\gamma_{1}^{2} \mathbf{I}, \\
\Lambda_{66}^{\prime} & =-\gamma_{1}^{2} \mathbf{I}, \\
\Lambda_{77}^{\prime} & =-\frac{15 n_{1}}{23 h_{1}} \mathbf{P} .
\end{aligned}
$$

Proof. Introducing the state transformation $\mathbf{x}(t)=e^{-\alpha_{1} t} \zeta(t)$, equations (1) and (6) can be transformed into the following forms:

$$
\left\{\begin{array}{l}
\dot{\zeta}(t)=\sum_{i=1}^{r} \xi_{i}(\theta(t))\left[\widetilde{A}_{i} \zeta(t)+\mathrm{B}_{i} \mathbf{u}_{\alpha_{1}}(t)+\mathbf{E}_{\mathbf{f i}} \mathbf{f}_{\alpha_{1}}(t)+\mathbf{E}_{\mathbf{w} i} \mathbf{w}_{\alpha_{1}}(t)\right], \\
\mathbf{y}_{\alpha_{1}}(t)=\sum_{i=1}^{r} \xi_{i}(\theta(t))\left[\mathbf{C}_{i} \zeta\left(t-\tau_{1}(t)\right)+\mathbf{E}_{\mathbf{v} i} \mathbf{v}_{\alpha_{1}}\left(t-\tau_{1}(t)\right)\right],
\end{array}\right.
$$

where

$$
\begin{aligned}
\widetilde{A}_{i} & =\mathbf{A}_{i}+\alpha_{1} \mathbf{I}, \\
\mathbf{u}_{\alpha_{1}}(t) & =e^{\alpha_{1} t} \mathbf{u}(t), \\
\mathbf{f}_{\alpha_{1}}(t) & =e^{\alpha_{1} t} \mathbf{f}(t), \\
\mathbf{w}_{\alpha_{1}}(t) & =e^{\alpha_{1} t} \mathbf{w}(t), \\
\mathbf{y}_{\alpha_{1}}(t) & =e^{\alpha_{1}\left(t-\tau_{1}(t)\right)} \mathbf{y}(t), \\
\mathbf{v}_{\alpha_{1}}\left(t-\tau_{1}(t)\right) & =e^{\alpha_{1}\left(t-\tau_{1}(t)\right)} \mathbf{v}\left(t-\tau_{1}(t)\right) .
\end{aligned}
$$

The state transformation $\hat{x}(t)=e^{-\alpha_{1} t} \hat{\zeta}(t)$ is also introduced to obtain an observer for system state and fault estimation with $\alpha$ - stability:

$$
\left\{\begin{array}{l}
\dot{\hat{\zeta}}_{(t)}=\sum_{i=1}^{r} \sum_{j=1}^{r} \xi_{i}(\theta(t)) \xi_{j}(\theta(t))\left\{\widetilde{A}_{i} \widehat{\zeta}(t)+\mathbf{B}_{i} \mathbf{u}_{\alpha_{1}}(t)+\mathbf{E}_{\mathbf{f i}} \widehat{f}_{\alpha_{1}}(t)-\mathbf{L}_{j} e^{\alpha_{1} \tau_{1}(t)}\left[\widehat{y}_{\alpha_{1}}(t)-\mathbf{y}_{\alpha_{1}}(t)\right]\right\} \\
\hat{y}_{\alpha_{1}}(t)=\sum_{i=1}^{r} \xi_{i}(\theta(t))\left\{\mathbf{C}_{i} \widehat{\zeta}\left(t-\tau_{1}(t)\right)\right\}, \\
\dot{\hat{f}}_{\alpha_{1}}(t)=\sum_{i=1}^{r} \xi_{j}(\theta(t))\left[-e^{\alpha_{1} \tau_{1}(t)} \mathbf{F}_{j} \mathbf{e}_{y_{\alpha_{1}}}(t)\right]
\end{array}\right.
$$

where $\widehat{\zeta}(t), \widehat{y}_{\alpha_{1}}(t)$, and $\widehat{f}_{\alpha_{1}}(t)$ are the estimations of system state, the observer output, and the actuator fault, respectively.
We define $\mathbf{e}_{y \alpha_{1}}(t)=\widehat{y}_{\alpha_{1}}(t)-\mathbf{y}_{\alpha_{1}}(t), \mathbf{e}_{f \alpha_{1}}(t)=\widehat{f}_{\alpha_{1}}(t)-$ $\mathbf{f}_{\alpha_{1}}(t)$, and then, the following error system can be obtained:

$$
\left\{\begin{array}{l}
\dot{e}_{x \alpha_{1}}(t)=\sum_{i=1}^{r} \sum_{j=1}^{r} \xi_{i}(\theta(t)) \xi_{j}(\theta(t))\left[\widetilde{A}_{i} \mathbf{e}_{x \alpha_{1}}(t)+\mathbf{E}_{\mathbf{f i}} \mathbf{e}_{f \alpha_{1}}(t)-\mathbf{E}_{\mathbf{w} i} \mathbf{w}_{\alpha_{1}}(t)-\mathbf{L}_{j} \mathbf{C}_{i} e^{\alpha_{1} \tau_{1}(t)} \mathbf{e}_{x \alpha_{1}}\left(t-\tau_{1}(t)\right)+\mathbf{L}_{j} \mathbf{E}_{\mathbf{v} i} e^{\alpha_{1} \tau_{1}(t)} \mathbf{v}_{\alpha_{1}}\left(t-\tau_{1}(t)\right)\right] \\
\mathbf{e}_{y \alpha_{1}}(t)=\sum_{i=1}^{r} \xi_{i}(\theta(t))\left[\mathbf{C}_{i} \mathbf{e}_{x \alpha_{1}}\left(t-\tau_{1}(t)\right)-\mathbf{E}_{\mathbf{v i}} \mathbf{v}_{\alpha_{1}}\left(t-\tau_{1}(t)\right)\right] .
\end{array}\right.
$$

The derivative of the fault estimation error with respect to time is

$$
\dot{e}_{f \alpha_{1}}(t)=\sum_{i=1}^{r} \sum_{j=1}^{r} \xi_{i}(\theta(t)) \xi_{j}(\theta(t))\left\{-e^{\alpha_{1} \tau_{1}(t)}\left[\mathbf{F}_{j} \mathbf{C}_{i} \mathbf{e}_{x \alpha_{1}}\left(t-\tau_{1}(t)\right)+\mathbf{F}_{j} \mathbf{E}_{\mathbf{v} i} \mathbf{v}_{\alpha_{1}}\left(t-\tau_{1}(t)\right)\right]-\dot{f}_{\alpha_{1}}(t)\right\} .
$$


The estimation errors of system state and fault are augmented into the following system:

$$
\dot{\bar{e}}_{\alpha_{1}}(t)=\sum_{i=1}^{r} \sum_{j=1}^{r} \xi_{i}(\theta(t)) \xi_{j}(\theta(t))\left[\bar{A}_{i} \bar{e}_{\alpha_{1}}(t)-\bar{E}_{\mathbf{w} i} \bar{w}_{\alpha_{1}}(t)-\bar{L}_{j} \bar{C}_{i} e^{\alpha_{1} \tau_{1}(t)} \bar{e}_{\alpha_{1}}\left(t-\tau_{1}(t)\right)+\bar{L}_{j} \mathbf{E}_{v i} \mathbf{e}^{\alpha_{1} \tau_{1}(t)} \mathbf{v}_{\alpha_{1}}\left(t-\tau_{1}(t)\right)\right]
$$

We define

$$
\begin{aligned}
\bar{A}_{i} & =\left[\begin{array}{cc}
\tilde{A}_{i} & \mathbf{E}_{\mathbf{f} i} \\
\mathbf{0} & \mathbf{0}
\end{array}\right], \\
\bar{e}_{\alpha_{1}}(t) & =\left[\begin{array}{c}
\mathbf{e}_{x \alpha_{1}}(t) \\
\mathbf{e}_{f \alpha_{1}}(t)
\end{array}\right], \\
\bar{C}_{i} & =\left[\begin{array}{c}
\mathbf{C}_{i} \\
\mathbf{0}
\end{array}\right], \\
\bar{L}_{j} & =\left[\begin{array}{c}
\mathbf{L}_{j} \\
\mathbf{F}_{j}
\end{array}\right], \\
\bar{w}_{\alpha_{1}}(t) & =\left[\begin{array}{c}
\mathbf{w}_{\alpha_{1}}(t) \\
\dot{f}_{\alpha_{1}}(t)
\end{array}\right], \\
\bar{E}_{\mathbf{w} i} & =\left[\begin{array}{cc}
\mathbf{E}_{\mathbf{w} i} & \mathbf{0} \\
\mathbf{0} & \mathbf{I}
\end{array}\right] .
\end{aligned}
$$

According to the definition of $\alpha$ - stability, when the system equation (24) is asymptotically stable, the augmented error system equation (10) has $\alpha$ - stability.

In order to ensure the system equation (24) is asymptotically stable, let $\bar{w}_{\alpha_{1}}(t)=0$ and $\mathbf{v}_{\alpha_{1}}\left(t-\tau_{1}(t)\right)=0$, and the following Lyapunov functional is constructed:

$$
\begin{aligned}
\mathbf{V}_{1}(t)= & \bar{e}_{\alpha_{1}}^{T}(t) \mathbf{P} \bar{e}_{\alpha_{1}}(t)+\left(h_{1}-\tau_{1}(t)\right) \boldsymbol{\varphi}_{1}^{T}(t) \mathbf{S} \varphi_{1}(t) \\
& +\left(h_{1}-\tau_{1}(t)\right) \tau_{1}(t) \bar{e}_{\alpha_{1}}^{T}\left(t-\tau_{1}(t)\right) \mathbf{Q} \bar{e}_{\alpha_{1}}\left(t-\tau_{1}(t)\right) \\
& +\left(h_{1}-\tau_{1}(t)\right) \int_{t-\tau_{1}(t)}^{t} \dot{\bar{e}}_{\alpha_{1}}^{T}(s) \mathbf{R} \dot{\bar{e}}_{\alpha_{1}}(s) \mathrm{d} s,
\end{aligned}
$$

where

$$
\begin{aligned}
\mathbf{P} & =\mathbf{P}^{T}>\mathbf{0}, \\
\mathbf{Q} & =\mathbf{Q}^{T}>\mathbf{0}, \\
\mathbf{R} & =\mathbf{R}^{T}>\mathbf{0}, \\
\mathbf{S} & =\mathbf{S}^{T}>\mathbf{0}, \\
\boldsymbol{\varphi}_{1}(t) & =\bar{e}_{\alpha_{1}}(t)-\bar{e}_{\alpha_{1}}\left(t-\tau_{1}(t)\right) .
\end{aligned}
$$

Derivation of $\mathbf{V}_{1}(t)$ along the system equation (24):

$$
\begin{aligned}
\dot{V}_{1}(t)= & 2 \bar{e}_{\alpha_{1}}^{T}(t) \mathbf{P} \dot{\bar{e}}_{\alpha_{1}}(t)-\int_{t-\tau_{1}(t)}^{t} \dot{\bar{e}}_{\alpha_{1}}^{T}(s) \mathbf{R} \dot{\bar{e}}_{\alpha_{1}}(s) \mathrm{d} s \\
& -h_{1} \bar{e}_{\alpha_{1}}^{T}\left(t-\tau_{1}(t)\right) \mathbf{Q} \bar{e}_{\alpha_{1}}\left(t-\tau_{1}(t)\right) \\
& +2\left(h_{1}-\tau_{1}(t)\right) \bar{e}_{\alpha_{1}}^{T}\left(t-\tau_{1}(t)\right) \mathbf{Q} \bar{e}_{\alpha_{1}}\left(t-\tau_{1}(t)\right) \\
& +\left(h_{1}-\tau_{1}(t)\right) \dot{\bar{e}}_{\alpha_{1}}^{T}(t) \mathbf{R} \dot{\bar{e}}_{\alpha_{1}}(t)-\boldsymbol{\varphi}_{1}^{T}(t) \mathbf{S} \boldsymbol{\varphi}_{1}(t) \\
& +2\left(h_{1}-\tau_{1}(t)\right) \boldsymbol{\varphi}_{1}^{T}(t) \mathbf{S} \dot{\bar{e}}_{\alpha_{1}}(t) .
\end{aligned}
$$

In order to deal with the integral term in equation (28), the affine Bessel-Legendre inequality in [44] is adopted; i.e.,

$$
-\int_{t-\tau_{1}(t)}^{t} \dot{\bar{e}}_{\alpha_{1}}^{T}(s) \mathbf{R} \dot{\bar{e}}_{\alpha_{1}}(s) \mathrm{d} s \leq-\psi_{1}^{T}(t) \Delta \psi_{1}(t)
$$

where

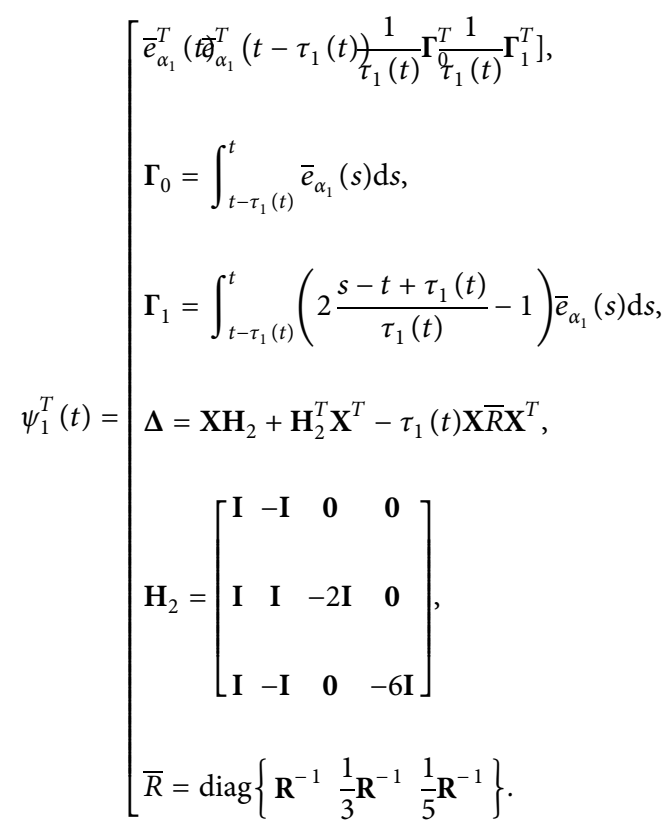

Substituting the inequality in equation (29) into equation (28), let

$$
\begin{aligned}
& \mathrm{N}_{11}=\left[\begin{array}{llll}
\mathbf{I} & \mathbf{0} & \mathbf{0} & 0
\end{array}\right] \text {, } \\
& \mathrm{N}_{12}=\left[\begin{array}{llll}
\bar{A}_{i} & -e^{\alpha_{1} \tau_{1}(t)} \bar{L}_{j} \bar{C}_{i} & \mathbf{0} & \mathbf{0}
\end{array}\right] \text {, } \\
& \mathrm{N}_{13}=\left[\begin{array}{llll}
\mathbf{0} & \text { I } & \mathbf{0} & 0
\end{array}\right] \text {, } \\
& \mathrm{N}_{14}=\left[\begin{array}{llll}
\mathbf{I} & -\mathbf{I} & \mathbf{0} & 0
\end{array}\right] \text {, }
\end{aligned}
$$


and then,

$$
\begin{aligned}
\bar{e}_{\alpha_{1}}(t) & =\mathrm{N}_{11} \psi_{1}(t), \\
\dot{\bar{e}}_{\alpha_{1}}(t) & =\mathrm{N}_{12} \psi_{1}(t), \\
\bar{e}_{\alpha_{1}}\left(t-\tau_{1}(t)\right) & =\mathrm{N}_{13} \psi_{1}(t), \\
\varphi_{1}(t) & =\mathrm{N}_{14} \psi_{1}(t),
\end{aligned}
$$

so

$$
\dot{V}_{1}(t) \leq \boldsymbol{\psi}_{1}^{T}(t)\left[\boldsymbol{\Omega}_{11}+\left(h_{1}-\tau_{1}(t)\right) \boldsymbol{\Omega}_{12}+\tau_{1}(t) \boldsymbol{\Omega}_{13}\right] \psi_{1}(t),
$$

where

$$
\begin{aligned}
& \boldsymbol{\Omega}_{11}=2 \mathrm{~N}_{11}^{T} \mathbf{P N}_{12}-h_{1} \mathbf{N}_{13}^{T} \mathbf{Q N} \mathbf{N}_{13}-\mathrm{N}_{14}^{T} \mathbf{S N}_{14}-\mathbf{X H}_{2}-\mathbf{H}_{2}^{T} \mathbf{X}^{T}, \\
& \boldsymbol{\Omega}_{12}=2 \mathbf{N}_{13}^{T} \mathbf{Q N}_{13}+\mathrm{N}_{12}^{T} \mathbf{R N}_{12}+2 \mathrm{~N}_{14}^{T} \mathbf{S N}_{12}, \\
& \boldsymbol{\Omega}_{13}=\mathbf{X} \bar{R} \mathbf{X}^{T} .
\end{aligned}
$$

When $\Omega_{11}+\left(h_{1}-\tau_{1}(t)\right) \Omega_{12}+\tau_{1}(t) \Omega_{13}<\mathbf{0}$ is established, according to Lyapunov stability theory, the system equation (24) is asymptotically stable; i.e., the augmented error system equation (10) has $\alpha$-stability.

Using the linear convex combinatorial lemma [45], it is obtained that the necessary and sufficient conditions for $\left(h_{1}-\tau_{1}(t)\right) \Omega_{12}+\tau_{1}(t) \Omega_{13}<\mathbf{0}$ are as follows:

$$
\begin{aligned}
& \boldsymbol{\Omega}_{11}+h_{1} \boldsymbol{\Omega}_{12}<\mathbf{0}, \\
& \boldsymbol{\Omega}_{11}+h_{1} \boldsymbol{\Omega}_{13}<\mathbf{0} .
\end{aligned}
$$

Under the zero initial condition, when $\mathbf{v}_{\alpha_{1}}\left(t-\tau_{1}(t)\right) \neq 0, \bar{w}_{\alpha_{1}}(t) \neq 0$, considering the $\mathbf{H}_{\infty}$ performance index function,

$$
\begin{aligned}
\mathbf{J}_{1} & =\dot{V}_{1}(t)+\bar{e}_{\alpha_{1}}^{T}(t) \bar{e}_{\alpha_{1}}(t) \\
& -\gamma_{1}^{2}\left[\bar{w}_{\alpha_{1}}^{T}(t) \bar{w}_{\alpha_{1}}(t)+\mathbf{v}_{\alpha_{1}}^{T}\left(t-\tau_{1}(t)\right) \mathbf{v}_{\alpha_{1}}\left(t-\tau_{1}(t)\right)\right]<0 .
\end{aligned}
$$

Let

$$
\begin{aligned}
& \psi_{2}^{T}(t)=\left[\bar{e}_{\alpha_{1}}^{T}(t) \bar{e}_{\alpha_{1}}^{T}\left(t-\tau_{1}(t)\right) \frac{1}{\tau_{1}(t)} \boldsymbol{\Gamma}_{0}^{T} \frac{1}{\tau_{1}(t)} \boldsymbol{\Gamma}_{1}^{T} \mathbf{v}_{\alpha_{1}}^{T}\left(t-\tau_{1}(t)\right) \bar{w}_{\alpha_{1}}^{T}(t)\right], \\
& \mathrm{N}_{21}=\left[\begin{array}{llllll}
\mathbf{I} & 0 & 0 & 0 & 0 & 0
\end{array}\right] \text {, } \\
& \mathrm{N}_{22}=\left[\begin{array}{lllll}
\bar{A}_{i} & -e^{\alpha_{1} \tau_{1}(t)} \bar{L}_{j} \bar{C}_{i} & \mathbf{0} & \mathbf{0} & e^{\alpha_{1} \tau_{1}(t)} \bar{L}_{j} \mathbf{E}_{\mathbf{v i}}-\bar{E}_{\mathbf{w} i}
\end{array}\right], \\
& \mathrm{N}_{23}=\left[\begin{array}{llllll}
0 & \text { I } & 0 & 0 & 0 & 0
\end{array}\right] \text {, } \\
& \mathrm{N}_{24}=\left[\begin{array}{llllll}
\mathbf{I} & -\mathbf{I} & \mathbf{0} & \mathbf{0} & \mathbf{0} & \mathbf{0}
\end{array}\right] \text {, } \\
& \mathrm{N}_{25}=\left[\begin{array}{llllll}
\text { I } & 0 & 0 & 0 & 0 & 0 \\
0 & \text { I } & 0 & 0 & 0 & 0 \\
0 & 0 & \text { I } & 0 & 0 & 0 \\
0 & 0 & 0 & \text { I } & 0 & 0
\end{array}\right], \\
& \mathrm{N}_{26}=\left[\begin{array}{llllll}
0 & 0 & 0 & 0 & \text { I } & 0 \\
0 & 0 & 0 & 0 & 0 & \text { I }
\end{array}\right],
\end{aligned}
$$

and then,

$$
\begin{aligned}
\bar{e}_{\alpha_{1}}(t) & =\mathrm{N}_{21} \psi_{2}(t), \\
\dot{\bar{e}}_{\alpha_{1}}(t) & =\mathrm{N}_{22} \psi_{2}(t), \\
\bar{e}_{\alpha_{1}}\left(t-\tau_{1}(t)\right) & =\mathrm{N}_{23} \psi_{2}(t), \\
\varphi_{1}(t) & \left.=\mathrm{N}_{24} \psi_{2}(t)\right), \\
\psi_{1}(t) & =\mathrm{N}_{25} \psi_{2}(t), \\
{\left[\mathbf{v}_{\alpha_{1}}^{T}\left(t-\tau_{1}(t)\right) \bar{w}_{\alpha_{1}}^{T}(t)\right]^{T} } & =\mathrm{N}_{26} \psi_{2}(t) .
\end{aligned}
$$

Therefore, the following can be obtained:

$$
\mathbf{J}_{1} \leq \boldsymbol{\psi}_{2}^{T}(t)\left[\boldsymbol{\Omega}_{21}+\left(h_{1}-\tau_{1}(t)\right) \boldsymbol{\Omega}_{22}+\tau_{1}(t) \boldsymbol{\Omega}_{23}\right] \boldsymbol{\psi}_{2}(t)<\mathbf{0},
$$

where

$$
\begin{aligned}
\boldsymbol{\Omega}_{21}= & 2 \mathrm{~N}_{21}^{T} \mathbf{P} \mathrm{N}_{22}-h_{1} \mathbf{N}_{23}^{T} \mathbf{Q N} \mathbf{N}_{23}-\mathbf{N}_{24}^{T} \mathbf{S N}_{24} \\
& -\gamma_{1}^{2} \mathbf{N}_{26}^{T} \mathbf{N}_{26}-\mathrm{N}_{25}^{T}\left(\mathbf{X H}_{2}+\mathbf{H}_{2}^{T} \mathbf{X}^{T}\right) \mathrm{N}_{25}+\mathrm{N}_{21}^{T} \mathrm{~N}_{21}, \\
\boldsymbol{\Omega}_{22}= & 2 \mathrm{~N}_{23}^{T} \mathbf{Q} \mathrm{N}_{23}+\mathrm{N}_{22}^{T} \mathbf{R N}_{22}+2 \mathrm{~N}_{24}^{T} \mathbf{S N}_{22}, \\
\boldsymbol{\Omega}_{23}= & \mathrm{N}_{25}^{T} \mathbf{X} \bar{R} \mathbf{X}^{T} \mathrm{~N}_{25} .
\end{aligned}
$$


Furthermore, according to the linear convex combination lemma, the necessary and sufficient conditions for $\Omega_{21}+$ $\left(h_{1}-\tau_{1}(t)\right) \Omega_{22}+\tau_{1}(t) \Sigma_{23}<\mathbf{0}$ are

$$
\begin{aligned}
& \boldsymbol{\Omega}_{21}+h_{1} \boldsymbol{\Omega}_{22}<\mathbf{0}, \\
& \boldsymbol{\Omega}_{21}+h_{1} \boldsymbol{\Omega}_{23}<\mathbf{0} .
\end{aligned}
$$

Let $\mathbf{R}=n_{1} \mathbf{P}, \mathbf{S}=n_{2} \mathbf{P}, \mathbf{Q}=n_{3} \mathbf{P}, \mathbf{P} \bar{L}_{j}=\mathbf{Y}_{j}$, the non-linear matrix inequalities in equations (35) and (41) can be changed into linear, and furthermore, equations (12)-(15) can be obtained by using the Schur complement lemma.

Integrating equation (36) from 0 to $+\infty$,

$$
\begin{aligned}
& \mathbf{V}_{1}(+\infty)-\mathbf{V}_{1}(0) \leq-\int_{0}^{+\infty} \bar{e}_{\alpha_{1}}^{T}(t) \bar{e}_{\alpha_{1}}(t) \mathrm{d} t+\gamma_{1}^{2} \\
& {\left[\int_{0}^{+\infty} \bar{w}_{\alpha_{1}}^{T}(t) \bar{w}_{\alpha_{1}}(t) \mathrm{d} t+\sum_{k_{1}=0}^{+\infty}\left(i_{k_{1}+1}-i_{k_{1}}\right) \mathbf{v}_{\alpha_{1}}^{T}\left(i_{k_{1}}\right) \mathbf{v}_{\alpha_{1}}\left(i_{k_{1}}\right)\right],}
\end{aligned}
$$

where $V_{1}(0)=0, V_{1}(+\infty) \geq 0, \bar{w}_{\alpha_{1}}(t) \in \mathbf{L}_{2}[0,+\infty)$.

$$
\mathbf{v}_{\alpha_{1}}\left(i_{k_{1}}\right) \in \mathbf{L}_{2}[0,+\infty) \text {; thus, }
$$

$$
\begin{aligned}
& \int_{0}^{+\infty} \bar{e}_{\alpha_{1}}^{T}(t) \bar{e}_{\alpha_{1}}(t) \mathrm{d} t \\
& \leq \gamma_{1}^{2}\left[\int_{0}^{+\infty} \bar{w}_{\alpha_{1}}^{T}(t) \bar{w}_{\alpha_{1}}(t) \mathrm{d} t+\sum_{k_{1}=0}^{+\infty}\left(i_{k_{1}+1}-i_{k_{1}}\right) \mathbf{v}_{\alpha_{1}}^{T}\left(i_{k_{1}}\right) \mathbf{v}_{\alpha_{1}}\left(i_{k_{1}}\right)\right] .
\end{aligned}
$$

So, $\quad\left\|\bar{e}_{\alpha_{1}}(t)\right\|_{2}^{2} \leq \gamma_{1}^{2}\left[\left\|\bar{w}_{\alpha_{1}}(t)\right\|_{2}^{2}+\sum_{k_{1}=0}^{+\infty}\left(i_{k_{1}+1}-i_{k_{1}}\right) \quad \| \mathbf{v}_{\alpha_{1}}\right.$ $\left.\left(i_{k_{1}}\right) \|_{2}^{2}\right]$ is established, and the relevant $\mathbf{H}_{\infty}$ performance index is verified.

Remark 4. As demonstrated in [44], although the addition of matrix $\mathbf{X}$ increases the decision variable and the computational complexity when affine Bessel-Legendre inequality deals with the integral term, the conservatism can be significantly reduced, which can increase the solution space and enhance the attack defense ability of the system.

\section{Dual Security Controller for a Non-Linear CPS with Multi-Objective Constraints under DoS Attack}

Design objective: under the DETCS, in order to maintain the closed-loop fault system is asymptotically stable and has $\alpha$-stability, the dual security controller gain and event trigger matrix are obtained cooperatively, and finally, the system has good dual security under the dual threat of DoS attacks and actuator fault, and network resources can be saved at the same time.

Under the DETCS, the non-uniform transmission period is transformed into time-varying delay $\tau_{2}(t)$, and taking into account the fault accommodation and the ability of attack tolerance, the dynamic output feedback security controller is adopted as follows:

$$
\mathbf{u}(t)=\sum_{i=1}^{r} \sum_{j=1}^{r} \xi_{i}(\theta(t)) \xi_{j}(\theta(t))\left[\mathbf{K}_{j} \widehat{x}\left(t-\tau_{2}(t)\right)-\mathbf{B}_{i}^{+} \mathbf{E}_{\mathbf{f i}} \widehat{f}\left(t-\tau_{2}(t)\right)\right]
$$

where $t \in\left[j_{k_{3}}, j_{k_{3}+1}\right)$, the dual security controller gain matrix is $\mathbf{K}_{j} \in \mathbf{R}^{n_{u} \times n}$, and $\mathbf{K}_{j} \widehat{x}\left(t-\tau_{2}(t)\right)$ is the dynamic output feedback term. The fault accommodation matrix $\mathbf{B}_{i}^{+} \in \mathbf{R}^{n_{u} \times n}$ satisfies $\left(\mathbf{I}-\mathbf{B}_{i} \mathbf{B}_{j}^{+}\right) \mathbf{E}_{\mathbf{f} i}=0, \operatorname{rank}\left(\mathbf{B}_{i}, \mathbf{E}_{\mathbf{f} i}\right)=\operatorname{rank}\left(\mathbf{B}_{i}\right)$, and $-\mathbf{B}_{i}^{+} \mathbf{E}_{\mathbf{f} i} \widehat{f}\left(t-\tau_{2}(t)\right)$ is the fault compensation term.

Substituting equation (44) into equation (1), a non-linear closed-loop CPS model integrating attack-tolerant and faulttolerant control can be obtained:

$$
\begin{aligned}
\dot{x}(t)= & \sum_{i=1}^{r} \sum_{j=1}^{r} \xi_{i}(\theta(t)) \xi_{j}(\theta(t))\left[\mathbf{A}_{i} \mathbf{x}(t)+\mathbf{B}_{i} \mathbf{K}_{j} \mathbf{x}\left(t-\tau_{2}(t)\right)\right. \\
& +\mathbf{B}_{i} \mathbf{K}_{j} \mathbf{e}_{x}\left(t-\tau_{2}(t)\right)-\mathbf{E}_{\mathbf{f} i} \mathbf{e}_{f}\left(t-\tau_{2}(t)\right) \\
& \left.+\mathbf{E}_{\mathbf{w} i} \mathbf{w}(t)+\tau_{2}(t) \mathbf{E}_{\mathbf{f} i} \dot{f}(t)\right]
\end{aligned}
$$

Theorem 2. Considering the non-linear CPS in equation (45) with actuator time-varying fault $\mathbf{f}(t)$ and DoS attacks, under the DETCS, given positive numbers $n_{i}, m_{i}, i=1,2,3$, $\alpha_{2}, h_{2}, \gamma_{2}, \delta_{s}$, if there are symmetric positive definite matrix $\widetilde{P}>\mathbf{0}$ and appropriate dimensioned matrices $\widetilde{X}, \mathbf{K}_{1 j}, \mathbf{Q}_{1}, \mathbf{Q}_{3}, \mathbf{Q}_{5}$, the following matrix inequalities hold:

$$
\left[\begin{array}{cccccc}
\boldsymbol{\Pi}_{11} & \boldsymbol{\Pi}_{12} & \boldsymbol{\Pi}_{13} & \boldsymbol{\Pi}_{14} & \mathbf{0} & \mathbf{0} \\
* & \boldsymbol{\Pi}_{22} & \boldsymbol{\Pi}_{23} & \boldsymbol{\Pi}_{24} & \boldsymbol{\Pi}_{25} & \boldsymbol{\Pi}_{26} \\
* & * & \boldsymbol{\Pi}_{33} & \boldsymbol{\Pi}_{34} & \mathbf{0} & \mathbf{0} \\
* & * & * & \boldsymbol{\Pi}_{44} & \mathbf{0} & \mathbf{0} \\
* & * & * & * & \boldsymbol{\Pi}_{55} & \mathbf{0} \\
* & * & * & * & * & \boldsymbol{\Pi}_{66}
\end{array}\right]<0
$$

$$
\left[\begin{array}{cccccc}
\boldsymbol{\Pi}_{11}^{\prime} & \boldsymbol{\Pi}_{12}^{\prime} & \boldsymbol{\Pi}_{13} & \boldsymbol{\Pi}_{14} & \mathbf{0} & \tilde{X} \\
* & \boldsymbol{\Pi}_{22}^{\prime} & \boldsymbol{\Pi}_{23} & \boldsymbol{\Pi}_{24} & \boldsymbol{\Pi}_{25} & \tilde{X} \\
* & * & \boldsymbol{\Pi}_{33} & \boldsymbol{\Pi}_{34} & \mathbf{0} & \tilde{X} \\
* & * & * & \boldsymbol{\Pi}_{44} & \mathbf{0} & \tilde{X} \\
* & * & * & * & \boldsymbol{\Pi}_{55} & \mathbf{0} \\
* & * & * & * & * & \boldsymbol{\Pi}_{66}^{\prime}
\end{array}\right]<0,
$$

$$
\begin{aligned}
& {\left[\begin{array}{cc}
\mathrm{T}_{11} & \mathrm{~T}_{12} \\
* & \mathrm{~T}_{22}
\end{array}\right]<0,} \\
& {\left[\begin{array}{cc}
\mathrm{T}_{11}^{\prime} & \mathrm{T}_{12}^{\prime} \\
* & \mathrm{~T}_{22}^{\prime}
\end{array}\right]<0,}
\end{aligned}
$$




$$
\begin{gathered}
{\left[\begin{array}{cc}
\mathbf{Q}_{1} & \mathbf{E}_{\mathbf{f} i}^{T} \widetilde{P} \\
* & m_{1} \widetilde{P}
\end{array}\right]>0,} \\
{\left[\begin{array}{cc}
\mathbf{Q}_{3} & n_{1} \mathbf{E}_{\mathbf{f} i}^{T} \widetilde{P} \\
* & m_{2} \widetilde{P}
\end{array}\right]>0,} \\
{\left[\begin{array}{cc}
\mathbf{Q}_{5} & n_{2} \mathbf{E}_{\mathrm{f} i}^{T} \widetilde{P} \\
* & m_{3} \widetilde{P}
\end{array}\right]>0 .}
\end{gathered}
$$

Then, the system equation (45) has $\alpha$-stability and meets the $\mathbf{H}_{\infty}$ performance index

$$
\begin{array}{r}
\|\boldsymbol{\eta}(t)\|_{2}^{2} \leq \gamma_{2}^{2}\left[\left\|\mathbf{w}_{\alpha_{2}}(t)\right\|_{2}^{2}+\sum_{k_{3}=0}^{+\infty}\left(j_{k_{3}+1}-j_{k_{3}}\right)\right. \\
\left.\left(\left\|\mathbf{e}_{x \alpha_{2}}\left(j_{k_{3}}\right)\right\|_{2}^{2}+\left\|\mathbf{e}_{f \alpha_{2}}\left(j_{k_{3}}\right)\right\|_{2}^{2}\right)\right],
\end{array}
$$

and dual security controller gain $\mathbf{K}_{j}=\left(\widetilde{P} \mathbf{B}_{i}\right)^{+} \mathbf{K}_{1 j}$ and trigger weight matrix $\Phi$ are obtained cooperatively.

Here,

$$
\begin{aligned}
\Pi_{11}= & \widetilde{P} \breve{A}_{i}+\breve{A}_{i}^{T} \widetilde{P}-n_{1} \widetilde{P}+\frac{h_{2}^{2}}{4} m_{3} \breve{A}_{i}^{T} \widetilde{P} \breve{A}_{i}+\frac{h_{2}^{2}}{4} m_{2} \widetilde{P} \\
& +h_{2} n_{2} \breve{A}_{i}^{T} \widetilde{P} \breve{A}_{i}+h_{2} n_{1}\left(\widetilde{P} \breve{A}_{i}+\breve{A}_{i}^{T} \widetilde{P}\right)-3 \widetilde{X}-3 \widetilde{X}^{T}, \\
\Pi_{12}= & e^{\alpha_{2} \tau_{2}(t)} \mathbf{K}_{1 j}+n_{1} \widetilde{P}+h_{2} n_{1} \mathbf{K}_{1 j}-h_{2} n_{1} \breve{A}_{i}^{T} \widetilde{P}+\widetilde{X}-3 \widetilde{X}^{T} \\
& +\frac{h_{2}^{2}}{4} m_{3} e^{\alpha_{2} \tau_{2}(t)} \breve{A}_{i}^{T} \mathbf{K}_{1 j}-\frac{h_{2}^{2}}{4} m_{2} \widetilde{P}+h_{2} n_{2} e^{\alpha_{2} \tau_{2}(t)} \breve{A}_{i}^{T} \mathbf{K}_{1 j}, \\
\Pi_{13}= & 2 \widetilde{X}-3 \widetilde{X}^{T}, \\
\Pi_{14}= & 6 \widetilde{X}-3 \widetilde{X}^{T}, \\
\Pi_{22}= & h_{2} n_{3} \widetilde{P}-n_{1} \widetilde{P}+\frac{h_{2}^{2}}{4} m_{2} \widetilde{P}-h_{2} n_{1}\left(\mathbf{K}_{1 j}+\mathbf{K}_{1 j}^{T}\right)+\widetilde{X}+\widetilde{X}^{T}, \\
\Pi_{23}= & 2 \widetilde{X}+\widetilde{X}^{T}, \\
\Pi_{24}= & 6 \widetilde{X}+\widetilde{X}^{T}, \\
\Pi_{25}= & \frac{h_{2}^{2}}{4} m_{3} e^{2 \alpha_{2} \tau_{2}(t)} \mathbf{K}_{1 j}^{T}, \\
\Pi_{26}= & h_{2} n_{2} e^{2 \alpha_{2} \tau_{2}(t)} \mathbf{K}_{1 j}^{T}, \\
\Pi_{33}= & 2 \widetilde{X}+2 \widetilde{X}^{T}, \\
\Pi_{34}= & 6 \widetilde{X}+2 \widetilde{X}^{T}, \\
\Pi_{44}= & 6 \widetilde{X}+6 \widetilde{X}^{T}, \\
\Pi_{55}= & -\frac{h_{2}^{2}}{4} m_{3} e^{2 \alpha_{2} \tau_{2}(t)} \widetilde{P}, \\
\Pi_{66}= & -h_{2} n_{2} e^{2 \alpha_{2} \tau_{2}(t)} \widetilde{P}, \\
\Pi_{11}^{\prime}= & \widetilde{P} \breve{A}_{i}+\breve{A}_{i}^{T} \widetilde{P}-n_{1} \widetilde{P}+\frac{h_{2}^{2}}{4} m_{3} \breve{A}_{i}^{T} \widetilde{P} \breve{A}_{i}+\frac{h_{2}^{2}}{4} m_{2} \widetilde{P} \\
& \\
&
\end{aligned}
$$

$$
\begin{aligned}
& \Pi_{12}^{\prime}=e^{\alpha_{2} \tau_{2}(t)} \mathbf{K}_{1 j}+n_{1} \widetilde{P}+\widetilde{X}-3 \widetilde{X}^{T} \\
& +\frac{h_{2}^{2}}{4} m_{3} e^{\alpha_{2} \tau_{2}(t)} \stackrel{-T}{A} \mathbf{K}_{1 j}-\frac{h_{2}^{2}}{4} m_{2} \widetilde{P} \\
& \Pi_{66}^{\prime}=-\frac{15 n_{2}}{23 h_{2}} \widetilde{P}, \\
& \Pi_{22}^{\prime}=-h_{2} n_{3} \widetilde{P}-n_{1} \widetilde{P}+\frac{h_{2}^{2}}{4} m_{2} \widetilde{P}+\widetilde{X}+\widetilde{X}^{T}, \\
& \Psi_{11}=\Pi_{11}+\mathbf{I} \text {, } \\
& \Psi_{12}=\Pi_{12} \text {, } \\
& \Psi_{13}=\Pi_{13} \text {, } \\
& \Psi_{14}=\Pi_{14} \text {, } \\
& \boldsymbol{\Psi}_{15}=e^{\alpha_{2} \tau_{2}(t)} \mathbf{K}_{1 j}+h_{2} n_{2} e^{\alpha_{2} \tau_{2}(t)} \stackrel{-T}{A_{i}^{T}} \mathbf{K}_{1 j}+h_{2} n_{1} e^{\alpha_{2} \tau_{2}(t)} \mathbf{K}_{1 j}, \\
& +\frac{h_{2}^{2}}{4} m_{3} e^{\alpha_{2} \tau_{2}(t)} \stackrel{-T}{A_{i}^{T}} \mathbf{K}_{1 j} \text {, } \\
& \Psi_{16}=-\frac{h_{2}^{2}}{4} m_{3} e^{\alpha_{2} \tau_{2}(t)} \stackrel{-}{A} \widetilde{P} \widetilde{P} \mathbf{E}_{\mathrm{f} i} \\
& -e^{\alpha_{2} \tau_{2}(t)} \widetilde{P} \mathbf{E}_{\mathbf{f} i}-h_{2} n_{2} e^{\alpha_{2} \tau_{2}(t)} \stackrel{A}{i}_{i}^{T} \widetilde{P} \mathbf{E}_{\mathrm{f} i}-h_{2} n_{1} e^{\alpha_{2} \tau_{2}(t)} \widetilde{P} \mathbf{E}_{\mathbf{f i}}, \\
& \mathrm{T}_{11}=\left[\begin{array}{cccccc}
\Psi_{11} & \boldsymbol{\Psi}_{12} & \boldsymbol{\Psi}_{13} & \boldsymbol{\Psi}_{14} & \boldsymbol{\Psi}_{15} & \boldsymbol{\Psi}_{16} \\
* & \boldsymbol{\Psi}_{22} & \boldsymbol{\Psi}_{23} & \boldsymbol{\Psi}_{24} & \boldsymbol{\Psi}_{25} & \boldsymbol{\Psi}_{26} \\
* & * & \boldsymbol{\Psi}_{33} & \boldsymbol{\Psi}_{34} & \mathbf{0} & \mathbf{0} \\
* & * & * & \boldsymbol{\Psi}_{44} & \mathbf{0} & \mathbf{0} \\
* & * & * & * & \boldsymbol{\Psi}_{55} & \boldsymbol{\Psi}_{56} \\
* & * & * & * & * & \boldsymbol{\Psi}_{66}
\end{array}\right], \\
& \mathrm{T}_{12}=\left[\begin{array}{ccccc}
\Psi_{17} & \mathbf{0} & \mathbf{0} & \mathbf{0} & \mathbf{0} \\
\boldsymbol{\Psi}_{27} & \mathbf{0} & \mathbf{0} & \mathbf{0} & h_{2} n_{2} e^{2 \alpha_{2} \tau_{2}(t)} \mathbf{K}_{1 j}^{T} \\
\mathbf{0} & \mathbf{0} & \mathbf{0} & \mathbf{0} & \mathbf{0} \\
\mathbf{0} & \mathbf{0} & \mathbf{0} & \mathbf{0} & \mathbf{0} \\
\boldsymbol{\Psi}_{57} & \mathbf{0} & \mathbf{0} & \mathbf{0} & h_{2} n_{2} e^{2 \alpha_{2} \tau_{2}(t)} \\
\boldsymbol{\Psi}_{67}^{T} & \mathbf{0} & \mathbf{0} & \mathbf{0} & \mathbf{0}
\end{array}\right],
\end{aligned}
$$

$$
\mathrm{T}_{22}=\left[\begin{array}{ccccc}
\boldsymbol{\Psi}_{77} & \mathbf{0} & \mathbf{0} & \mathbf{0} & \mathbf{0} \\
* & \sigma_{s} \boldsymbol{\Phi} & \mathbf{0} & \mathbf{0} & \mathbf{0} \\
* & * & -\boldsymbol{\Phi} & \mathbf{0} & \mathbf{0} \\
* & * & * & \mathbf{0} & \mathbf{0} \\
* & * & * & * & -h_{2} n_{2} e^{2 \alpha_{2} \tau_{2}(t)} \widetilde{P}
\end{array}\right],
$$$$
\boldsymbol{\Psi}_{17}=\widetilde{P} \mathbf{B}_{\mathbf{w} i}+\frac{h_{2}^{2}}{4} m_{3} \stackrel{\sim}{A}_{i}^{T} \widetilde{P} \mathbf{E}_{\mathbf{w} i}+h_{2} n_{1} \widetilde{P} \mathbf{E}_{\mathbf{w} i}
$$$$
\Psi_{22}=h_{2} n_{3} \widetilde{P}-n_{1} \widetilde{P}+\widetilde{X}+\widetilde{X}^{T}+\frac{h_{2}^{2}}{4} m_{2} \widetilde{P}
$$$$
-h_{2} n_{1} e^{\alpha_{2} \tau_{2}(t)}\left(\mathbf{K}_{1 j}+\mathbf{K}_{1 j}^{T}\right) \text {, }
$$

$\Psi_{23}=\Pi_{23}$,

$\Psi_{24}=\Pi_{24}$,

$\Psi_{25}=-h_{2} n_{1} e^{\alpha_{2} \tau_{2}(t)} \mathbf{K}_{1 j}$,

$\boldsymbol{\Psi}_{26}=h_{2} n_{1} e^{\alpha_{2} \tau_{2}(t)} \widetilde{P} \mathbf{E}_{\mathbf{f} i}-\frac{h_{2}^{2}}{4} m_{3} e^{2 \alpha_{2} \tau_{2}(t)} \mathbf{K}_{1 j}^{T} \mathbf{E}_{\mathbf{f} i}$ 


$$
\begin{aligned}
& -h_{2} n_{2} e^{2 \alpha_{2} \tau_{2}(t)} \mathbf{K}_{1 j}^{T} \mathbf{E}_{\mathbf{f} i}, \\
& \boldsymbol{\Psi}_{27}=-\frac{h_{2}^{2}}{4} m_{3} e^{\alpha_{2} \tau_{2}(t)} \mathbf{K}_{1 j}^{T} \mathbf{E}_{\mathbf{w} i} \text {, } \\
& -h_{2} n_{2} e^{\alpha_{2} \tau_{2}(t)} \mathbf{K}_{1 j}^{T} \mathbf{E}_{\mathbf{w} i}-h_{2} n_{1} \widetilde{P} \mathbf{E}_{\mathbf{w} i}, \\
& \Psi_{33}=\Pi_{33} \text {, } \\
& \Psi_{34}=\Pi_{34} \text {, } \\
& \Psi_{44}=\Pi_{44} \text {, } \\
& \Psi_{55}=-\gamma_{2}^{2} \mathbf{I} \text {, } \\
& \boldsymbol{\Psi}_{56}=-\frac{h_{2}^{2}}{4} m_{3} e^{2 \alpha_{2} \tau_{2}(t)} \mathbf{K}_{1 j}^{T} \mathbf{E}_{\mathbf{f} i}-h_{2} n_{2} e^{2 \alpha_{2} \tau_{2}(t)} \mathbf{K}_{1 j}^{T} \mathbf{E}_{\mathbf{f} i}, \\
& \boldsymbol{\Psi}_{57}=\frac{h_{2}^{2}}{4} m_{3} e^{\alpha_{2} \tau_{2}(t)} \mathbf{K}_{1 j}^{T} \mathbf{E}_{\mathbf{w} i}+h_{2} n_{2} e^{\alpha_{2} \tau_{2}(t)} \mathbf{K}_{1 j}^{T} \mathbf{E}_{\mathbf{w} i}, \\
& \boldsymbol{\Psi}_{66}=\frac{h_{2}^{2}}{4} m_{3} e^{2 \alpha_{2} \tau_{2}(t)} \mathbf{E}_{\mathbf{f} i}^{T} \widetilde{P} \mathbf{E}_{\mathbf{f} i}+h_{2} n_{2} e^{2 \alpha_{2} \tau_{2}(t)} \mathbf{E}_{\mathbf{f} i}^{T} \widetilde{P} \mathbf{E}_{\mathbf{f} i}-\gamma_{2}^{2} \mathbf{I}, \\
& \Psi_{67}=-\frac{h_{2}^{2}}{4} m_{3} e^{\alpha_{2} \tau_{2}(t)} \mathbf{E}_{\mathbf{f} i}^{T} \widetilde{P} \mathbf{E}_{\mathbf{w} i}-h_{2} n_{2} e^{\alpha_{2} \tau_{2}(t)} \mathbf{E}_{\mathbf{f} i}^{T} \widetilde{P} \mathbf{E}_{\mathbf{w} i}, \\
& \boldsymbol{\Psi}_{77}=-\gamma_{2}^{2} \mathbf{I}+\frac{h_{2}^{2}}{4} m_{3} \mathbf{E}_{\mathbf{w} i}^{T} \widetilde{P} \mathbf{E}_{\mathbf{w} i}+h_{2} n_{2} \mathbf{E}_{\mathbf{w} i}^{T} \widetilde{P} \mathbf{E}_{\mathbf{w} i} \text {, } \\
& \mathrm{T}_{11}^{\prime}=\left[\begin{array}{cccccc}
\boldsymbol{\Psi}_{11}^{\prime} & \boldsymbol{\Psi}_{12}^{\prime} & \boldsymbol{\Psi}_{13}^{\prime} & \boldsymbol{\Psi}_{14}^{\prime} & \boldsymbol{\Psi}_{15}^{\prime} & \boldsymbol{\Psi}_{16}^{\prime} \\
* & \boldsymbol{\Psi}_{22}^{\prime} & \boldsymbol{\Psi}_{23}^{\prime} & \boldsymbol{\Psi}_{24}^{\prime} & \mathbf{0} & \boldsymbol{\Psi}_{26}^{\prime} \\
* & * & \boldsymbol{\Psi}_{33}^{\prime} & \boldsymbol{\Psi}_{34}^{\prime} & \mathbf{0} & \mathbf{0} \\
* & * & * & \boldsymbol{\Psi}_{44}^{\prime} & \mathbf{0} & \mathbf{0} \\
* & * & * & * & \boldsymbol{\Psi}_{55}^{\prime} & \boldsymbol{\Psi}_{56}^{\prime} \\
* & * & * & * & * & \boldsymbol{\Psi}_{66}^{\prime}
\end{array}\right], \\
& \mathrm{T}_{12}^{\prime}=\left[\begin{array}{ccccc}
\Psi_{17}^{\prime} & \mathbf{0} & \mathbf{0} & \tilde{X} & \mathbf{0} \\
\boldsymbol{\Psi}_{27}^{\prime} & \mathbf{0} & \mathbf{0} & \tilde{X} & \frac{h_{2}^{2}}{4} m_{3} e^{2 \alpha_{2} \tau_{2}(t)} \mathbf{K}_{1 j}^{T} \\
\mathbf{0} & \mathbf{0} & \mathbf{0} & \tilde{X} & \mathbf{0} \\
\mathbf{0} & \mathbf{0} & \mathbf{0} & \tilde{X} & \mathbf{0} \\
\Psi_{57}^{\prime} & \mathbf{0} & \mathbf{0} & \mathbf{0} & \frac{h_{2}^{2}}{4} m_{3} e^{2 \alpha_{2} \tau_{2}(t)} \mathbf{K}_{1 j}^{T} \\
\Psi_{67}^{\prime} & \mathbf{0} & \mathbf{0} & \mathbf{0} & \mathbf{0}
\end{array}\right],
\end{aligned}
$$$$
\mathrm{T}_{22}^{\prime}=\left[\begin{array}{ccccc}
\Psi_{77}^{\prime} & \mathbf{0} & \mathbf{0} & \mathbf{0} & \mathbf{0} \\
* & \sigma_{s} \boldsymbol{\Phi} & \mathbf{0} & \mathbf{0} & \mathbf{0} \\
* & * & -\boldsymbol{\Phi} & \mathbf{0} & \mathbf{0} \\
* & * & * & -\frac{15 n_{2}}{23 h_{2}} \widetilde{P} & \mathbf{0} \\
* & * & * & * & -\frac{h_{2}^{2}}{4} m_{3} e^{2 \alpha_{2} \tau_{2}(t)} \widetilde{P}
\end{array}\right],
$$

$$
\begin{aligned}
& \Psi_{11}^{\prime}=\Pi_{11}^{\prime}+\mathbf{I}, \\
& \Psi_{12}^{\prime}=\Pi_{12}^{\prime} \text {, } \\
& \Psi_{13}^{\prime}=\Pi_{13} \text {, } \\
& \Psi_{14}^{\prime}=\Pi_{14} \text {, } \\
& \boldsymbol{\Psi}_{15}^{\prime}=e^{\alpha_{2} \tau_{2}(t)} \mathbf{K}_{1 j}+\frac{h_{2}^{2}}{4} m_{3} e^{\alpha_{2} \tau_{2}(t)} \stackrel{-T}{A_{i}^{T}} \mathbf{K}_{1 j}, \\
& \boldsymbol{\Psi}_{16}^{\prime}=-e^{\alpha_{2} \tau_{2}(t)} \widetilde{P} \mathbf{E}_{\mathbf{f} i} \\
& -\frac{h_{2}^{2}}{4} m_{3} e^{\alpha_{2} \tau_{2}(t)} \stackrel{-T}{A_{i}^{T}} \widetilde{P} \mathbf{E}_{\mathbf{f} i}, \\
& \mathbf{\Psi}_{17}^{\prime}=\widetilde{P} \mathbf{E}_{\mathbf{w} i}+\frac{h_{2}^{2}}{4} m_{3} \stackrel{A_{i}^{T}}{P} \widetilde{P} \mathbf{E}_{\mathbf{w} i} \\
& \Psi_{22}^{\prime}=-h_{2} n_{3} \widetilde{P}-n_{1} \widetilde{P}+\widetilde{X}+\widetilde{X}^{T}+\frac{h_{2}^{2}}{4} m_{2} \widetilde{P}, \\
& \Psi_{23}^{\prime}=\Pi_{23} \text {, } \\
& \Psi_{24}^{\prime}=\Pi_{24} \text {, } \\
& \boldsymbol{\Psi}_{26}=-\frac{h_{2}^{2}}{4} m_{3} e^{2 \alpha_{2} \tau_{2}(t)} \mathbf{K}_{1 j}^{T} \mathbf{E}_{\mathbf{f} i}, \\
& \Psi_{27}^{\prime}=-\frac{h_{2}^{2}}{4} m_{3} e^{\alpha_{2} \tau_{2}(t)} \mathbf{K}_{1 j}^{T} \mathbf{E}_{\mathbf{w} i}, \\
& \Psi_{33}^{\prime}=\Pi_{33} \text {, } \\
& \Psi_{34}^{\prime}=\Pi_{34}, \\
& \Psi_{44}^{\prime}=\Pi_{44}, \\
& \Psi_{55}^{\prime}=\Pi_{55} \text {, } \\
& \boldsymbol{\Psi}_{56}^{\prime}=-\frac{h_{2}^{2}}{4} m_{3} e^{2 \alpha_{2} \tau_{2}(t)} \mathbf{K}_{1 j}^{T} \mathbf{E}_{\mathbf{f} i}, \\
& \boldsymbol{\Psi}_{57}^{\prime}=\frac{h_{2}^{2}}{4} m_{3} e^{\alpha_{2} \tau_{2}(t)} \mathbf{K}_{1 j}^{T} \mathbf{E}_{\mathbf{w} i}, \\
& \Psi_{66}=-\gamma_{2}^{2} \mathbf{I}+\frac{h_{2}^{2}}{4} m_{3} e^{2 \alpha_{2} \tau_{2}(t)} \mathbf{E}_{\mathbf{f} i}^{T} \widetilde{P} \mathbf{E}_{\mathbf{f} i}, \\
& \Psi_{67}^{\prime}=-\frac{h_{2}^{2}}{4} m_{3} e^{\alpha_{2} \tau_{2}(t)} \mathbf{E}_{\mathbf{f} i}^{T} \widetilde{P} \mathbf{E}_{\mathbf{w} i} \\
& \boldsymbol{\Psi}_{77}^{\prime}=-\gamma_{2}^{2} \mathbf{I}+\frac{h_{2}^{2}}{4} m_{3} \mathbf{E}_{\mathbf{w} i}^{T} \widetilde{P} \mathbf{E}_{\mathbf{w} i} .
\end{aligned}
$$

Proof. The proof of Theorem 2 is similar to Theorem 1, and it will not be elaborated here.

Remark 5. When the performance index $\alpha_{2}$ and $\gamma_{2}$ are limited, the maximum allowable delay $h_{2}^{m}$ can be obtained by the following formula:

$$
\begin{gathered}
\max _{\widetilde{P}, \widetilde{X}, \mathbf{K}_{1}, \mathbf{Q}_{1}, \mathbf{Q}_{3}, \mathbf{Q}_{5}, \alpha_{2}, \gamma_{2}} h_{2}^{m} \\
\text { s.t. } \widetilde{P}>\mathbf{0},(46),(47),(48),(49),(50) .
\end{gathered}
$$

Furthermore, the maximum allowable consecutive packet losses under DoS attacks can be obtained through equation (5). 


$$
\tau_{M a}^{D o S}=\left\lfloor\frac{h_{2}^{m}-h_{t_{k_{2}}}^{\max }}{h_{t_{k_{2}}}^{\max }}\right\rfloor,
$$

where \lfloor\rfloor is the downward rounding symbol and $\tau_{M a}^{D o S}$ is the upper bound of the maximum consecutive packet losses caused by DoS attack.

\section{Strategy and Algorithm of DoS Attack Detection and Active Attack Tolerance}

5.1. Strategy of Attack Detection and Attack Tolerance. After the maximum allowable time delay $h_{2}^{m}$ is obtained through equation (58), in the process of data transmission, it is assumed that the control quantities $\mathbf{u}\left(j_{k_{3}} h\right)$ and $\mathbf{u}\left(j_{k_{3}+1} h\right)$ are transmitted to the intelligent execution unit at instant $j_{k_{3}} h$ and $j_{k_{3}+1} h$, respectively, and set $h_{j_{k_{3}}}=j_{k_{3}+1} h-j_{k_{3}} h$ as the transmission interval of two consecutive received control quantities. In order to ensure that the non-linear CPS has a certain security margin when DoS attack occurs, the security factor $\beta$ is set, and $\beta \in(0,1)$.

When $h_{j_{k_{3}}}<\beta h_{2}^{m}$, the non-linear CPS is not subject to DoS attack or is subject to a low-energy DoS attack, and it is sufficient to implement a passive attack-tolerance strategy at this time. When $h_{j_{k_{3}}} \geq \beta h_{2}^{m}$, it is detected that the duration of DoS attack is close to or exceeds the maximum allowed time delay; i.e., this is a high-energy DoS attack intrusion. The passive attack-tolerant strategy can no longer meet the system requirements, and a more targeted active attacktolerant strategy needs to be adopted. The strategy of DoS attack detection and attack-tolerance is shown in Figure 3.

5.2. Active Attack-Tolerant Algorithm for DoS Attack. At instant $j_{k_{3}+1} h$, the latest control quantities stored in the data buffer of the intelligent execution unit are $\mathbf{u}\left(j_{k_{3}} h\right)$ and $\mathbf{u}\left(j_{k_{3}-1} h\right)$. Whether the current control quantity $\mathbf{u}\left(j_{k_{3}+1} h\right)$ is transmitted or corrected depends on whether a DoS attack is high energy.

Once $h_{j_{k_{3}}} \geq \beta h_{2}^{m}$, it means that high-energy DoS attack invaded the system, and the current control quantity is blocked from transmission. At this time, if the system can compensate for the lack of control quantity caused by highenergy attack based on the previously stored in the data buffer, then the system can tolerate high-energy DoS attack in an active way.

Based on the PID control idea, the following is adopted to correct and compensate the current control quantity $\mathbf{u}\left(j_{k_{3}+1} h\right)$ in real time:

$$
\mathbf{u}\left(j_{k_{3}+1} h\right)=\mathbf{u}\left(j_{k_{3}} h\right)+k_{p}\left[\mathbf{u}\left(j_{k_{3}} h\right)-\mathbf{u}\left(j_{k_{3}-1} h\right)\right] .
$$

Through equation (60), the loss of control quantity caused by DoS attack can be corrected and compensated, so as to realize the active attack tolerance. The specific algorithm of active attack tolerance for high-energy DoS attacks is shown in Table 1.
Remark 6. In equation (60), the first term $\mathbf{u}\left(j_{k_{3}} h\right)$ represents the control quantity transmitted successfully at the last time, and the second term $k_{p}\left[\mathbf{u}\left(j_{k_{3}} h\right)-\mathbf{u}\left(j_{k_{3}-1} h\right)\right]$ represents the change of the control quantity successfully transmitted for two consecutive times, where $k_{p}$ is the proportion coefficient. The selection of $k_{p}$ directly affects the state of the control quantity and system performance, so it is necessary to select it reasonably.

Remark 7. If $h_{j_{k}} \geq \beta h_{2}^{m}$, then the CPS suffers from a highenergy DoS attack, and the lack of control quantity caused by the high-energy attack is updated with the help of the correction compensation algorithm in equation (60) based on the PID idea, so as to achieve active attack tolerance for the high-energy DoS attack. If $h_{j_{k_{3}}}<\beta h_{2}^{m}$, then it is a lowenergy DoS attack, and the received control quantity will be sent directly to the actuator and act on the controlled plant to passively tolerate attack in a resilient and robust manner. The introduction of security factor $\beta$ enables a seamless integration of the active and passive attack-tolerance strategy, so as to achieve a more effective defense against limited-energy DoS attacks.

\section{Simulation Research and Result Analysis}

6.1. Example Description. A classic quadruple-tank simulation example in [46] is used to verify the effectiveness of the results in this study, where

$$
\begin{aligned}
& \mathbf{A}_{1}=\left[\begin{array}{cccc}
-0.016 & 0 & 0.042 & 0 \\
0 & -0.011 & 0 & 0.033 \\
0 & 0 & -0.042 & 0 \\
0 & 0 & 0 & -0.033
\end{array}\right] \text {, } \\
& \mathbf{A}_{2}=\left[\begin{array}{cccc}
-0.022 & 0 & 0.061 & 0 \\
0 & -0.018 & 0 & 0.049 \\
0 & 0 & -0.064 & 0 \\
0 & 0 & 0 & -0.049
\end{array}\right] \text {, } \\
& \mathbf{A}_{3}=\left[\begin{array}{cccc}
-0.031 & 0 & 0.053 & 0 \\
0 & -0.021 & 0 & 0.067 \\
0 & 0 & -0.083 & 0 \\
0 & 0 & 0 & -0.061
\end{array}\right] \text {, } \\
& \mathbf{A}_{4}=\left[\begin{array}{cccc}
-0.039 & 0 & 0.106 & 0 \\
0 & -0.0276 & 0 & 0.0826 \\
0 & 0 & -0.107 & 0 \\
0 & 0 & 0 & -0.0827
\end{array}\right] \text {, } \\
& \mathbf{B}_{1}=\left[\begin{array}{cc}
0.083 & 0 \\
0 & 0.063 \\
0 & 0.048 \\
0.031 & 0
\end{array}\right] \text {, }
\end{aligned}
$$




$$
\begin{aligned}
& \mathbf{B}_{2}=\left[\begin{array}{cc}
0.1246 & 0 \\
0 & 0.093 \\
0 & 0.071 \\
0.045 & 0
\end{array}\right] \text {, } \\
& \mathbf{B}_{3}=\left[\begin{array}{cc}
0.165 & 0 \\
0 & 0.125 \\
0 & 0.097 \\
0.063 & 0
\end{array}\right] \text {, } \\
& \mathbf{B}_{4}=\left[\begin{array}{cc}
0.2076 & 0 \\
0 & 0.1576 \\
0 & 0.13 \\
0.0776 & 0
\end{array}\right] \text {, } \\
& \mathbf{E}_{\omega 1}=\left[\begin{array}{c}
0.01 \\
0.01 \\
0 \\
0.01
\end{array}\right], \mathbf{E}_{\omega 2}=\left[\begin{array}{c}
0.016 \\
0.016 \\
0 \\
0.016
\end{array}\right] \text {, } \\
& \mathbf{E}_{\omega 3}=\left[\begin{array}{c}
0.02 \\
0.02 \\
0 \\
0.02
\end{array}\right], \mathbf{E}_{\omega 4}=\left[\begin{array}{c}
0.024 \\
0.024 \\
0 \\
0.024
\end{array}\right] \text {, } \\
& \mathbf{E}_{f 1}=-\left[\begin{array}{llll}
0.083 & 0 & 0 & 0.031
\end{array}\right]^{T}, \\
& \mathbf{E}_{f 2}=-\left[\begin{array}{llll}
0.1246 & 0 & 0 & 0.0464
\end{array}\right]^{T}, \\
& \mathbf{E}_{f 3}=-\left[\begin{array}{llll}
0.167 & 0 & 0 & 0.061
\end{array}\right]^{T}, \\
& \mathbf{E}_{f 4}=-\left[\begin{array}{llll}
0.2076 & 0 & 0 & 0.0774
\end{array}\right]^{T}, \\
& \mathbf{C}_{1}=\operatorname{diag}\left\{\begin{array}{llll}
0.5 & 0.5 & 0.5 & 0.5
\end{array}\right\}, \\
& \mathbf{C}_{2}=\operatorname{diag}\left\{\begin{array}{llll}
0.48 & 0.48 & 0.48 & 0.48
\end{array}\right\}, \\
& \mathbf{C}_{3}=\operatorname{diag}\left\{\begin{array}{llll}
0.46 & 0.46 & 0.46 & 0.46
\end{array}\right\}, \\
& \mathbf{C}_{4}=\operatorname{diag}\left\{\begin{array}{llll}
0.52 & 0.52 & 0.52 & 0.52
\end{array}\right\}, \\
& \mathbf{E}_{v 1}=\left[\begin{array}{llll}
0.015 & 0 & 0.015 & 0.015
\end{array}\right]^{T}, \\
& \mathbf{E}_{v 2}=\left[\begin{array}{llll}
0.0224 & 0 & 0.0224 & 0.0224
\end{array}\right]^{T}, \\
& \mathbf{E}_{v 3}=\left[\begin{array}{llll}
0.030 & 0 & 0.025 & 0.027
\end{array}\right]^{T}, \\
& \mathbf{E}_{v 4}=\left[\begin{array}{llll}
0.0374 & 0 & 0.031 & 0.0326
\end{array}\right]^{T}, \\
& f(t)= \begin{cases}0, & t \leq 200, \\
2+2 \sin 0.01 \pi(t-100), & 200<t \leq 800 .\end{cases}
\end{aligned}
$$

The system disturbance $\mathbf{w}(t)$ and noise $\mathbf{v}\left(i_{k}\right)$ obey the independent white noise process, $\mathbf{N}(0.1,0.01)$, the initial state is $\mathbf{x}(0)=\left[\begin{array}{llll}4 & 4 & 2 & 2\end{array}\right]^{T}$, and the sampling period is $h=0.1 \mathrm{~s}$. Let $n_{1}=0.01, n_{2}=18, n_{3}=0.2, \gamma_{1}=2.7, \alpha_{1}=0.05$, according to Theorem 1 , the observer gain matrix $\mathbf{L}_{j}$ and fault estimation gain matrix $\mathbf{F}_{j}$ are obtained as follows:

$$
\begin{aligned}
\mathbf{L}_{1} & =\left[\begin{array}{cccc}
9.5113 & 3.5349 & -4.1339 & -2.4718 \\
0.0136 & 8.4589 & -0.0698 & -0.0059 \\
1.0766 & 3.4778 & 4.1001 & -2.1848 \\
1.1366 & 3.5093 & -4.7315 & 6.5135
\end{array}\right], \\
\mathbf{L}_{2} & =\left[\begin{array}{cccc}
9.7161 & 4.4964 & -8.0880 & -0.0016 \\
0.0152 & 8.4591 & -0.0601 & 0.0170 \\
1.3895 & 4.6108 & 2.8128 & -2.7352 \\
1.1995 & 4.5500 & -6.7495 & 7.0760
\end{array}\right], \\
\mathbf{L}_{3} & =\left[\begin{array}{cccc}
10.0753 & 5.9208 & -10.4613 & -0.1115 \\
0.0325 & 8.3092 & -0.0655 & 0.0021 \\
1.2245 & 4.7889 & 2.9127 & -3.0578 \\
1.2248 & 5.2170 & -7.5728 & 6.7892
\end{array}\right], \\
\mathbf{L}_{4}= & {\left[\begin{array}{llll}
8.1833 & 4.6009 & -9.9365 & 1.1258 \\
0.0294 & 6.2444 & -0.0747 & 0.0237 \\
0.7383 & 3.5723 & 1.8274 & -1.8031 \\
0.7586 & 3.7818 & -6.8698 & 6.5342
\end{array}\right], } \\
\mathbf{F}_{1}= & {\left[\begin{array}{llll}
-39.7821 & 3.7636 & 172.2936 & -131.9049
\end{array}\right], } \\
\mathbf{F}_{2}= & {\left[\begin{array}{llll}
-25.1256 & 7.2979 & 184.2543 & -163.6605
\end{array}\right], } \\
\mathbf{F}_{4}= & {\left[\begin{array}{llll}
-19.3933 & -4.4336 & 196.1008 & -158.0372
\end{array}\right], }
\end{aligned}
$$

In Theorem 2, we set $n_{1}=0.6, n_{2}=2, n_{3}=0.01, \gamma_{2}=3.2$, $\sigma_{s}=0.001, \alpha_{2}=0.06, m_{1}=m_{2}=m_{3}=0.2, h_{2}=2.4 s$, and the matrices $\Phi$ and $\mathbf{K}_{j}$ are obtained cooperatively according to Theorem 2.

$$
\begin{aligned}
& \boldsymbol{\Phi}=\operatorname{diag}\left\{\begin{array}{llll}
241.9598 & 241.9598 & 241.9598 & 241.9598
\end{array}\right\}, \\
& \mathbf{K}_{1}=\left[\begin{array}{cccc}
-30.3849 & 14.5296 & -24.0429 & 1.6240 \\
9.1219 & -36.4748 & 9.3747 & -18.1854
\end{array}\right] \text {, } \\
& \mathbf{K}_{2}=\left[\begin{array}{cccc}
-25.1803 & 11.5594 & -18.6989 & 1.2964 \\
7.4759 & -29.7709 & 7.2379 & -14.4292
\end{array}\right] \text {, } \\
& \mathbf{K}_{3}=\left[\begin{array}{cccc}
-15.5510 & 7.3717 & -12.5899 & 1.1794 \\
4.8103 & -17.9664 & 4.9429 & -10.0849
\end{array}\right] \text {, } \\
& \mathbf{K}_{4}=\left[\begin{array}{cccc}
-14.0035 & 7.1503 & -11.7226 & 1.5742 \\
4.1782 & -16.8248 & 4.4879 & -9.6635
\end{array}\right] \text {. }
\end{aligned}
$$

Then, the maximum allowable delay $h_{2}^{m}=2.592 \mathrm{~s}$ is obtained through equation (58), and the corresponding maximum number of consecutive packet losses is $\tau_{M a}^{D o S}=5$.

6.2. Estimations of State and Actuator Fault. In order to make the system stable operation with a certain attack-tolerance security margin, taking the security factor $\beta=0.9$, and combining with $h_{j_{k_{3}}} \geq \beta h_{2}^{m}$ in DoS attack detection algorithm, $h_{j_{k_{3}}}=2.33 \mathrm{~s}$. According to equation (58), the maximum number of consecutive packet losses within the corresponding security margin is $\tau_{M}^{D o S}=4$. If the number of packet losses is greater than the allowed maximum value, i.e., 


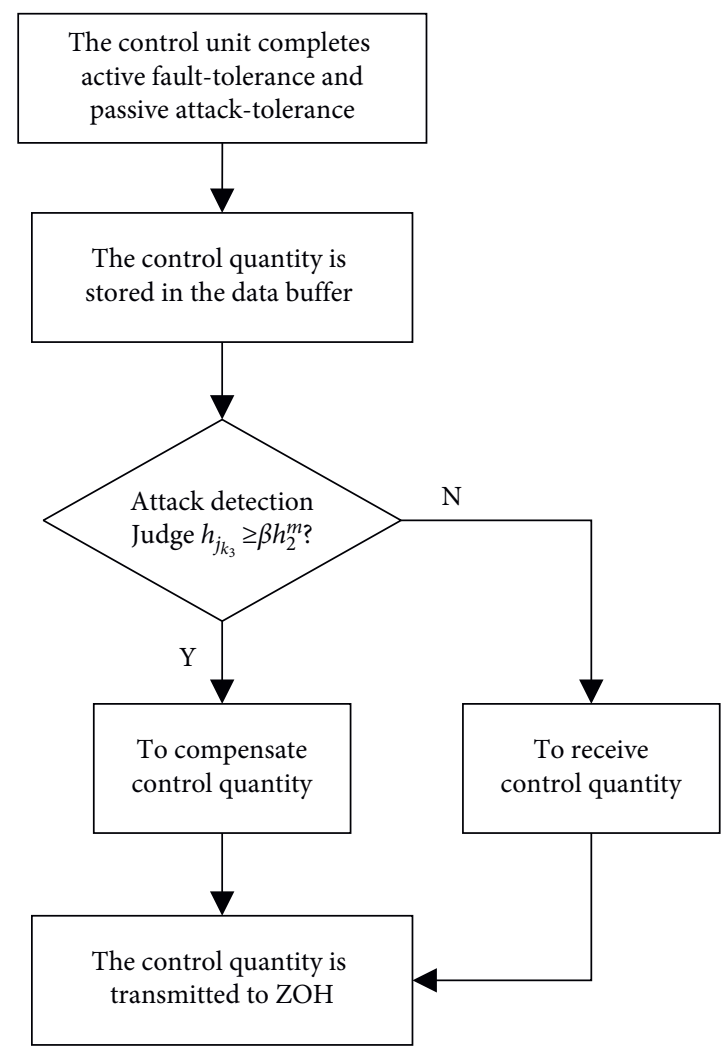

FIGURE 3: Schematic diagram of the strategy of DoS attack detection and attack-tolerance.

TABLE 1: Algorithm of DoS attack detection and control quantity compensation.

Step 1: the time interval between two consecutive received control quantities is obtained, and it is assumed that the transmission interval is $h_{j_{k_{3}}}=j_{k_{3}+1} h-j_{k_{3}} h$

Step 2: we judge $h_{j_{k_{3}}} \geq \beta h_{2}^{m}$ ?

Step 3: if Step 2 is true, the control quantity is corrected and compensated online according to (60); otherwise, Step 4 is directly executed

Step 4: the control quantity $\mathbf{u}\left(j_{k_{3}+1} h\right)$ is output at instant $j_{k_{3}+1} h$

Step 5: we return to Step 1

$\tau_{M}^{D o S}=7$ and $h_{2}^{m}=3.2 \mathrm{~s}$, then the DoS attack is of high energy. The sequence of DoS attack is shown in Figure 4, where 0 means no attack and 1 means there is an attack.

In order to compare the effectiveness of the proposed method, this paper compares it with the passive attacktolerant strategy using the same deduction process in [13]. For the convenience of subsequent analysis, it is specified that the active-passive attack-tolerant and active fault-tolerant control strategy in this study is dual security control strategy I, and the passive attack-tolerant and active faulttolerant strategy in [13] is dual security control strategy II. When dual security control strategies I and II are adopted, respectively, the curves of system state estimation errors, continuous time-varying fault estimation, fault estimation error, and system output are obtained, as shown in Figures 5-8.

In Figures 5(a), 6(a), and 7(a), let $k_{p}=1.3$, and dual security control strategy $I$ is adopted to deal with highenergy DoS attacks. The system states and actuator fault estimation can remain stable. The reason is that the activepassive attack-tolerant method corrects and compensates the control quantity that cannot be transmitted because the system delay exceeds the maximum allowable value. Therefore, the system defense capability is effectively improved.

When dual security control strategy II is adopted, the state estimation errors, continuous time-varying fault estimation, and error are as shown in Figures 5(b), 6(b), and $7(\mathrm{~b})$, respectively. It can be seen that the state errors, actuator fault estimation, and error begin to fluctuate after $300 \mathrm{~s}$, and the maximum fluctuation has exceeded \pm 2 . It shows that when the actual time delay is greater than the maximum allowable value, i.e., the system suffers from DoS attack with high energy, dual security control strategy II has been unable to effectively cope with high-energy DoS attack and actuator fault at this time. If the time delay continues to increase, the system fluctuation will further increase and even become unstable. 


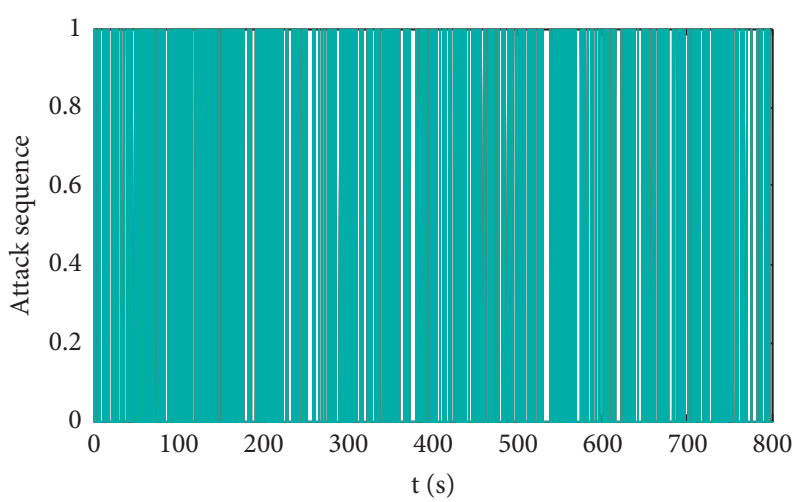

FIGURE 4: Sequence of DoS attack.

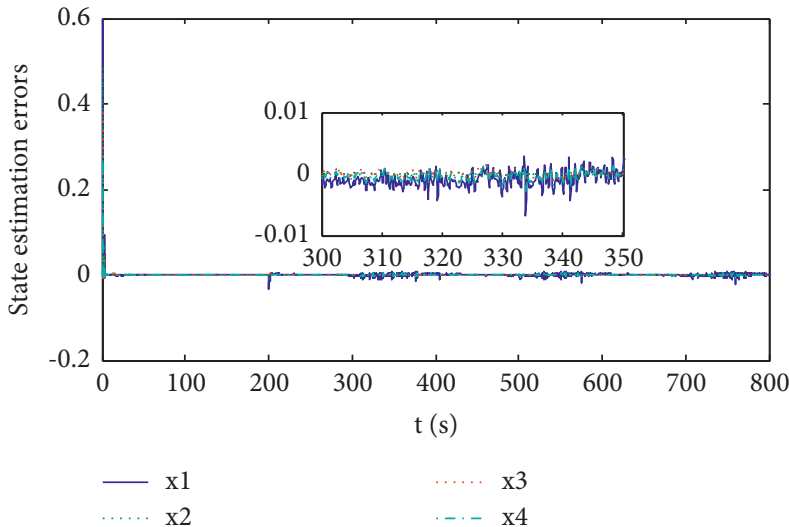

(a)

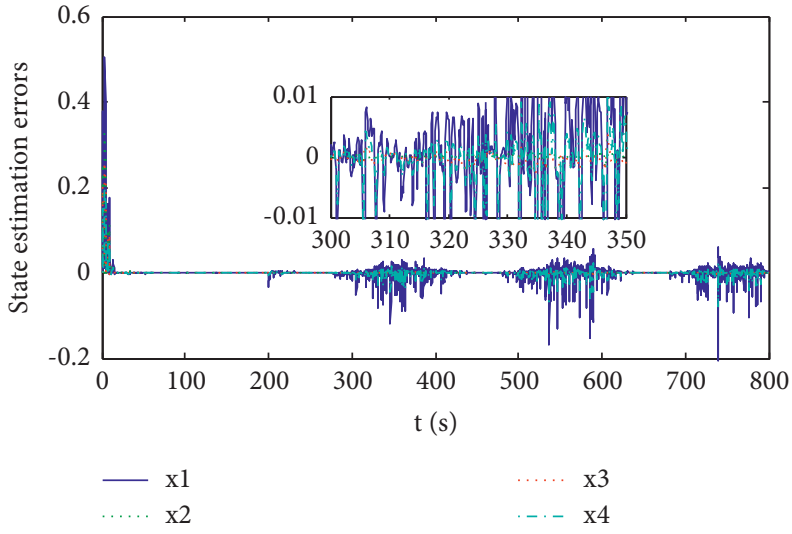

(b)

FiguRE 5: State estimation errors under different attack-tolerance strategies. (a) Dual security control strategy I and (b) dual security control strategy II.

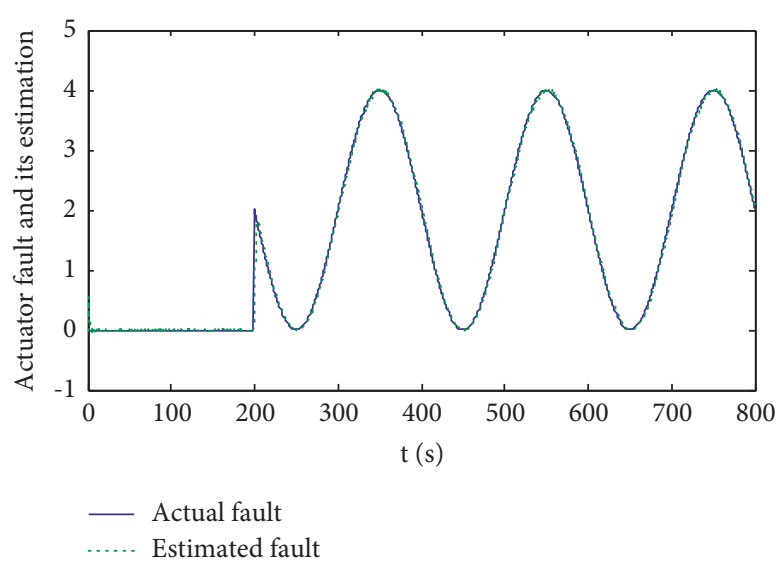

(a)

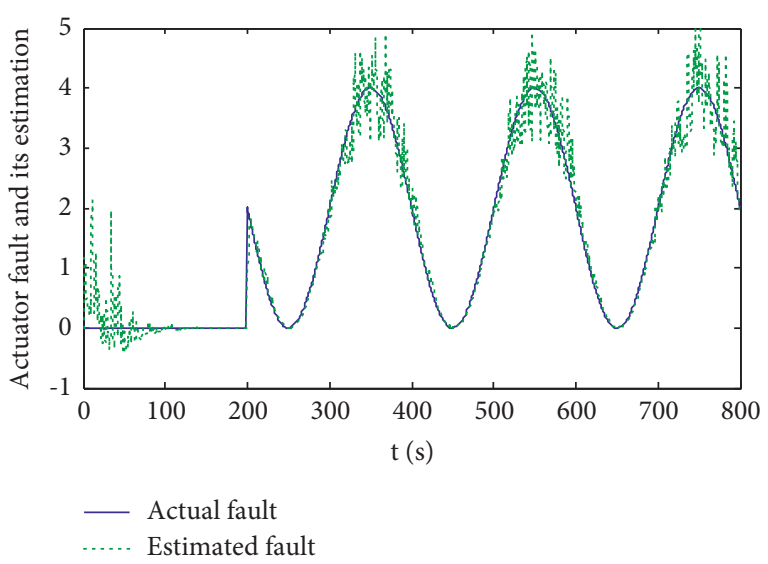

(b)

Figure 6: Actuator fault and its estimation under different attack-tolerance strategies. (a) Dual security control strategy I and (b) dual security control strategy II.

6.3. Analysis of the Trigger Parameter on System Security Performance. Compared with Figures 8(a) and 8(b), when dual security control strategy I is adopted, even if the system suffers from high-energy DoS attack and actuator fault, the system output remains stable at about $300 \mathrm{~s}$, while the system begins to fluctuate at $300 \mathrm{~s}$ when dual security control strategy II is adopted. In order to collaboratively analyse the dual security and communication resources, the data transmission amount and system security state under different trigger parameters are given in Table 2. 


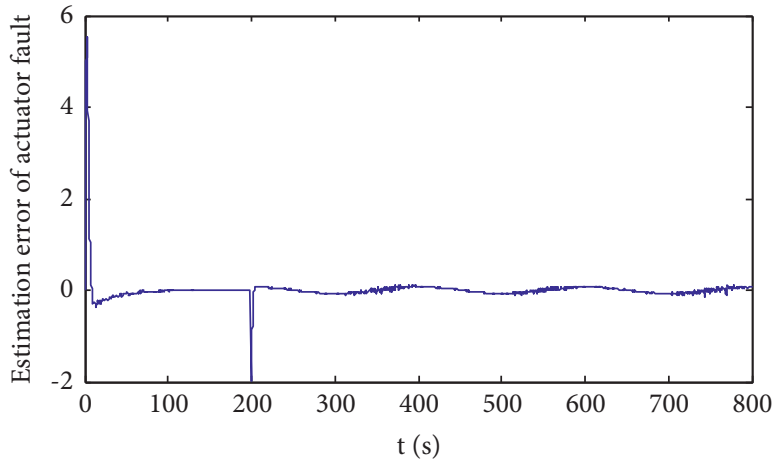

(a)

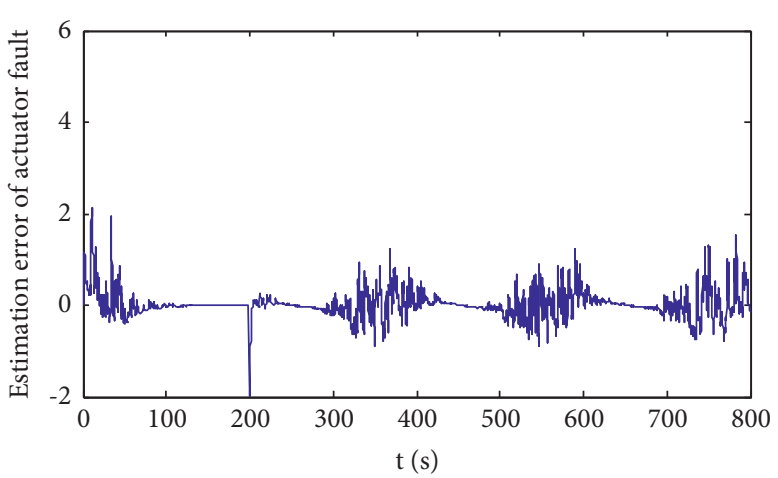

(b)

FIGURE 7: Actuator fault estimation error under different attack-tolerance strategies. (a) Dual security control strategy I and (b) dual security control strategy II.

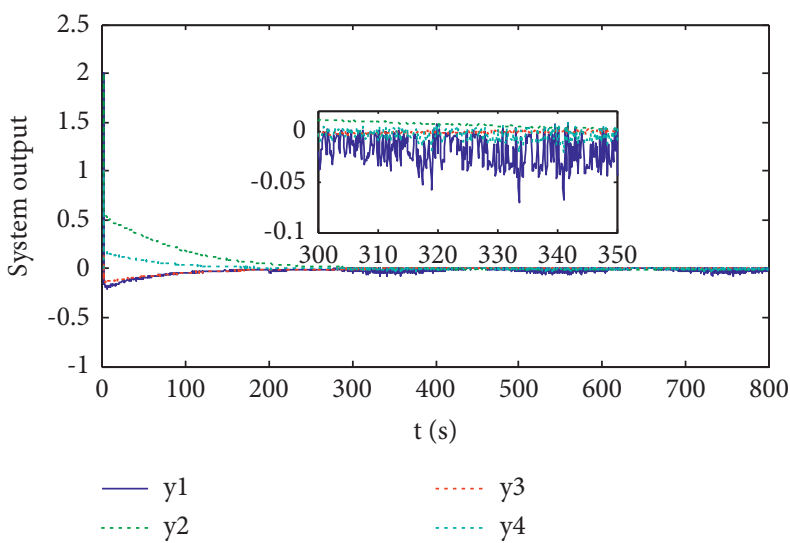

(a)

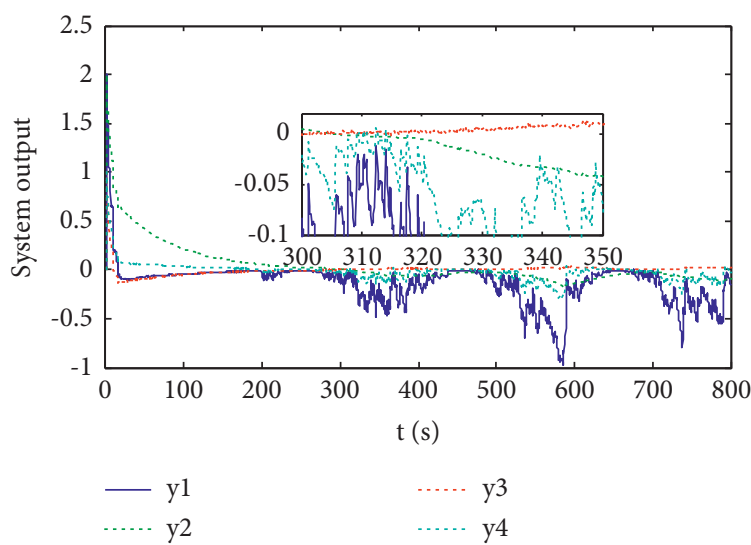

(b)

Figure 8: System output response under different attack-tolerance strategies. (a) Dual security control strategy I and (b) dual security control strategy II.

TABle 2: Data transmission amount and system security state under different trigger parameters.

\begin{tabular}{lcccc}
\hline$\delta_{s}$ & $n$ & $\bar{n}(\%)$ & $\bar{h}(\mathrm{~s})$ & System state \\
\hline 0 & 5672 & 70.9 & 0.141 & Stable \\
0.0004 & 4059 & 50.73 & 0.197 & Stable \\
0.0007 & 3641 & 45.51 & 0.22 & Stable \\
0.001 & 3163 & 39.54 & 0.253 & Stable \\
0.0013 & 2907 & 36.34 & 0.275 & Unstable \\
\hline
\end{tabular}

$n$ is the data transmission amount, $\bar{n}$ is the data transmission rate, and $\bar{h}$ is the average data transmission period.

On the one hand, with the increase of trigger parameter $\delta_{s}$, the amount of data transmission $n$ and the corresponding transmission rate $\bar{n}$ decrease, and the average transmission period $\bar{h}$ increases, but the system has become unstable. The continuous increase of $\delta_{s}$ helps to save network resources, but it is at the expense of system security. Therefore, by balancing the relationship between system security and network resources, the trigger parameter $\delta_{s}=0.001$ is finally selected in the study. On the other hand, when the trigger parameter is $\delta_{s}=0$, i.e., the periodic time trigger communication scheme is adopted, the transmitted data amount is
5672, while under the DETCS, the data transmission amount is 3163 . Obviously, the DETCS greatly reduces the amount of data transmission and effectively saves network resources.

\section{Conclusions}

A codesign of dual security control and communication for a non-linear CPS with actuator fault and DoS attack under the DETCS is studied, and the effectiveness of the proposed method is verified by a practical engineering case of a quadruple-tank system. The conclusions are as follows: 
(1) In order to effectively distinguish DoS attacks with different energy levels, an attack detection method is designed based on the maximum allowable delay. In addition, an active attack-tolerant strategy is proposed based on the PID idea for the lack of control quantity caused by high-energy DoS attack, which can effectively defend against high-energy DoS attack.

(2) Under multi-objective constraints, the design methods of an observer for state and fault and a controller with passive attack tolerance and active fault tolerance are given, respectively, which can effectively tolerate DoS attack with low energy and actuator fault. The methods can not only effectively save network resources but also have better dynamic performance, so as to improve the dual security control capability of the system.

(3) The active-passive attack-tolerance strategy expands the defense range of DoS attacks, and the introduction of security factor makes the intersection between active and passive attack tolerance, and it further improves the security degree of system attack tolerance.

In this study, the classical discrete event-triggered communication scheme is used, and although the network resources have been saved, the security and communication performance still need to be further optimized. In the future, more effective research on attack tolerance, fault tolerance, and communication resource saving will be carried out based on the adaptive event-triggered scheme. In addition, it is challenging to apply machine learning algorithms to effectively reconstruct and compensate for the data loss caused by DoS attacks.

\section{Data Availability}

The data used to support the findings of this study are included within the article.

\section{Conflicts of Interest}

The authors declare no conflicts of interest related to this article.

\section{Acknowledgments}

This study was financially supported by the National Natural Science of China (Grant nos. 62163022 and 61763027), Higher Education Innovation Capacity Improvement Project of Gansu in China (Grant no. 2019B-152), and Youth Science and Technology Fund of Gansu Province (Grant nos. 21JR1RM339 and 21JR7RM192).

\section{References}

[1] Y. Tang, Q. Chen, M. Y. Li, Q. Wang, M. Ni, and Y. Liang, "Overview on cyber-attacks against cyber physical power system," Automation of Electric Power Systems, vol. 40, no. 17, pp. 59-69, 2016.
[2] C. Adams, "Learning the lessons of WannaCry," Computer Fraud \& Security, vol. 2018, no. 9, pp. 6-9, 2018.

[3] H. Ge, D. Yue, X. Xie, S. Deng, and C. Dou, "A unified modeling of muti-sources cyber-attacks with uncertainties for CPS security control," Journal of the Franklin Institute, vol. 358, no. 1, pp. 89-113, 2021.

[4] S. M. Dibaji, M. Pirani, D. B. Flamholz, A. M. Annaswamy, K. H. Johansson, and A. Chakrabortty, "A systems and control perspective of CPS security," Annual Reviews in Control, vol. 47, pp. 394-411, 2019.

[5] A. Humayed, J. Lin, F. Li, and B. Luo, "Cyber-physical systems security-a survey," IEEE Internet of Things Journal, vol. 4, no. 6, pp. 1802-1831, 2017.

[6] M. Wolf and D. Serpanos, "Safety and security in cyberphysical systems and internet-of-things systems," Proceedings of the IEEE, vol. 106, no. 1, pp. 9-20, 2018.

[7] R. M. Czekster, C. Morisset, J. A. Clark, S. Soudjani, C. Patsios, and P. Davison, "Systematic review of features for co-simulating security incidents in cyber-physical systems," Security and Privacy, vol. 4, no. 3, 2021.

[8] R. Ma, P. Shi, Z. Wang, and L. Wu, "Resilient filtering for cyber-physical systems under denial-of-service attacks," International Journal of Robust and Nonlinear Control, vol. 30, no. 5, pp. 1754-1769, 2020.

[9] A.-Y. Lu and G.-H. Yang, "Stability analysis for cyber-physical systems under denial-of-service attacks," IEEE Transactions on Cybernetics, vol. 51, no. 11, pp. 5304-5313, 2021.

[10] F. Li and Y. Tang, "False data injection attack for cyberphysical systems with resource constraint," IEEE Transactions on Cybernetics, vol. 50, no. 2, pp. 729-738, 2020.

[11] D. Ye and T. Y. Zhang, "Summation detector for false datainjection attack in cyber-physical systems," IEEE Transactions on Cybernetics, vol. 50, no. 6, pp. 2338-2345, 2021.

[12] T. Liu, J. Tian, J. Z. Wang et al., "Integrated security threats and defense of cyber-physical systems," Acta Automatica Sinica, vol. 45, no. 1, pp. 5-24, 2019.

[13] L. Zhao and W. Li, "Co-design of dual security control and communication for nonlinear CPS under DoS attack," IEEE Access, vol. 8, pp. 19271-19285, 2020.

[14] S. Lai, B. Chen, T. Li, and L. Yu, "Packet-based state feedback control under DoS attacks in cyber-physical systems," IEEE Transactions on Circuits and Systems II: Express Briefs, vol. 66, no. 8, pp. 1421-1425, 2019.

[15] S. Feng and P. Tesi, "Resilient control under denial-of- service: robust design," Automatica, vol. 79, pp. 42-51, 2016.

[16] T. Li, B. Chen, L. Yu, and W.-A. Zhang, "Active security control approach against DoS attacks in cyber-physical systems," IEEE Transactions on Automatic Control, vol. 66, no. 9, pp. 4303-4310, 2021.

[17] S. Hu, D. Yue, X. Xie, X. Chen, and X. Yin, "Resilient eventtriggered controller synthesis of networked control systems under periodic DoS jamming attacks," IEEE Transactions on Cybernetics, vol. 49, no. 12, pp. 4271-4281, 2019.

[18] H. Yang, M. Shi, Y. Xia, and P. Zhang, "Security research on wireless networked control systems subject to jamming attacks," IEEE Transactions on Cybernetics, vol. 49, no. 6, pp. 2022-2031, 2019.

[19] G. K. Befekadu, V. Gupta, and P. J. Antsaklis, "Risk-sensitive control under Markov modulated denial-of-service (DoS) attack strategies," IEEE Transactions on Automatic Control, vol. 60, no. 12, pp. 3299-3304, 2015.

[20] S. Y. Lai, B. Chen, and L. Yu, "Switching-Luenberger -observer-based redundant control under DoS attacks," Control Theory \& Applications, vol. 37, no. 4, pp. 65-73, 2020. 
[21] M. Wang, Y. Liu, and B. Xu, "Observer-based $H_{\infty}$ control for cyber-physical systems encountering DoS jamming attacks: an attack-tolerant approach," ISA Transactions, vol. 104, pp. 1-14, 2020.

[22] J. Li and T. Li, "Cyber-physical security analysis of smart grids with Bayesian sequential game models," Acta Automatica Sinica, vol. 45, no. 1, pp. 98-109, 2019.

[23] Y. Huang and J. Zhao, "Cyber-physical systems with multiple denial-of-service attackers: a game-theoretic framework," IEEE Transactions on Circuits and Systems I: Regular Papers, vol. 68 , no. 10 , pp. 4349-4359, 2021.

[24] Q. Sun, K. Zhang, and Y. Shi, "Resilient model predictive control of cyber-physical systems under DoS attacks," IEEE Transactions on Industrial Informatics, vol. 16, no. 7, pp. 4920-4927, 2020.

[25] Z. W. Wang and W. Liu, "Event-triggered generalized predictive control of cyber-physical systems under denial-ofservice attacks," Journal of Shanghai Jiaotong University, vol. 54, no. 9, pp. 910-915, 2020.

[26] H. F. He, Y. Chen, W. H. Qi, M. L. Wang, and X. M. Chen, "Observer-based resilient control of positive systems with heterogeneous DoS attacks: a Markov model approach," Journal of the Franklin Institute, vol. 359, no. 1, pp. 272-293, 2021.

[27] P.-B. Wang, X.-M. Ren, and D.-D. Zheng, "Event-triggered resilient control for cyber-physical systems under periodic DoS jamming attacks," Information Sciences, vol. 577, pp. 541-556, 2021.

[28] Z.-H. Zhang, D. Liu, C. Deng, and Q.-Y. Fan, “A dynamic event-triggered resilient control approach to cyber-physical systems under asynchronous DoS attacks," Information Sciences, vol. 519, pp. 260-272, 2020.

[29] Y.-C. Sun and G.-H. Yang, "Periodic event-triggered resilient control for cyber-physical systems under denial-of-service attacks," Journal of the Franklin Institute, vol. 355, no. 13, pp. 5613-5631, 2018.

[30] X.-M. Zhang, Q.-L. Han, X. Ge, and L. Ding, "Resilient control design based on a sampled-data model for a class of networked control systems under denial-of-service attacks," IEEE Transactions on Cybernetics, vol. 50, no. 8, pp. 36163626, 2020.

[31] Z. H. Huo, Z. X. Zhang, and H. J. Fang, "Research on faulttolerant control of networked control systems based on information scheduling," Journal of Systems Engineering and Electronics, vol. 19, no. 5, pp. 1024-1028, 2008.

[32] J. Lan and R. J. Patton, "A new strategy for integration of fault estimation within fault-tolerant control," Automatica, vol. 69, pp. 48-59, 2016.

[33] J. Wang and M. M. Ling, "Hybrid robust $\mathrm{H}_{1} / \mathrm{H}_{\infty}$ fault-tolerant control with random delay NCS," Journal of Control Science and Engineering, vol. 2020, Article ID 1328068, 13 pages, 2020.

[34] B. Wang, B. Zhang, and R. Su, "Optimal tracking cooperative control for cyber-physical systems: dynamic Fault-tolerant control and resilient management," IEEE Transactions on Industrial Informatics, vol. 17, no. 1, pp. 158-167, 2021.

[35] C. Qian and H. Du, "Global output feedback stabilization of a class of nonlinear systems via linear sampled-data control," IEEE Transactions on Automatic Control, vol. 57, no. 11, pp. 2934-2939, 2012.

[36] C. Peng and T. C. Yang, "Event-triggered communication and $H_{\infty}$ control co-design for networked control systems," Automatica, vol. 49, no. 5, pp. 1326-1332, 2013.
[37] S. Hu, D. Yue, Q.-L. Han, X. Xie, X. Chen, and C. Dou, "Observer-based event-triggered control for networked linear systems subject to denial-of-service attacks," IEEE Transactions on Cybernetics, vol. 50, no. 5, pp. 1952-1964, 2020.

[38] R. Pan, Y. Tan, D. Du, and S. Fei, "Adaptive event-triggered synchronization control for complex networks with quantization and cyber-attacks," Neurocomputing, vol. 382, pp. 249-258, 2020.

[39] L. Zhang, H. Liang, Y. Sun, and C. K. Ahn, "Adaptive eventtriggered fault detection scheme for semi-Markovian jump systems with output quantization," IEEE Transactions on Systems, Man, and Cybernetics: Systems, vol. 51, no. 4, pp. 2370-2381, 2021.

[40] T. Takagi and M. Sugeno, "Fuzzy identification of systems and its applications to modeling and control," IEEE Transactions on Systems, Man, and Cybernetics, vol. SMC-15, no. 1, pp. 116-132, 1985.

[41] H. Q. Xiao, Y. He, M. Wu, and S. P. Xiao, "Output tracking control for sampled-data networked control systems in T-S fuzzy model," Acta Automatica Sinica, vol. 3, pp. 661-668, 2015.

[42] E. Fridman, "A refined input delay approach to sampled-data control," Automatica, vol. 46, no. 2, pp. 421-427, 2010.

[43] A. B. Qiu, H. G. Ji, and J. P. Gu, "Optimal integrated design of time-varying fault estimation and accommodation for noun uniformly sampled data systems," Acta Automatica Sinica, vol. 40, no. 7, pp. 1493-1504, 2014.

[44] W. I. Lee, S. Y. Lee, and P. Park, "Affine Bessel-Legendre inequality: application to stability analysis for systems with time-varying delays," Automatica, vol. 93, pp. 535-539, 2018.

[45] P. Park, J. W. Ko, and C. Jeong, "Reciprocally convex approach to stability of systems with time-varying delays," Automatica, vol. 47, no. 1, pp. 235-238, 2011.

[46] K. H. Johansson, "The quadruple-tank process: a multivariable laboratory process with an adjustable zero," IEEE Transactions on Control Systems Technology, vol. 8, no. 3, pp. 456-465, 2000. 